\title{
Protein-protein binding site identification by enumerating the configurations
}

\author{
Fei Guo ${ }^{1,2}$, Shuai Cheng $\mathrm{Li}^{2}$, Lusheng Wang ${ }^{2^{*}}$ and Daming Zhu
}

\begin{abstract}
Background: The ability to predict protein-protein binding sites has a wide range of applications, including signal transduction studies, de novo drug design, structure identification and comparison of functional sites. The interface in a complex involves two structurally matched protein subunits, and the binding sites can be predicted by identifying structural matches at protein surfaces.

Results: We propose a method which enumerates "all" the configurations (or poses) between two proteins (3D coordinates of the two subunits in a complex) and evaluates each configuration by the interaction between its components using the Atomic Contact Energy function. The enumeration is achieved efficiently by exploring a set of rigid transformations. Our approach incorporates a surface identification technique and a method for avoiding clashes of two subunits when computing rigid transformations. When the optimal transformations according to the Atomic Contact Energy function are identified, the corresponding binding sites are given as predictions. Our results show that this approach consistently performs better than other methods in binding site identification.

Conclusions: Our method achieved a success rate higher than other methods, with the prediction quality improved in terms of both accuracy and coverage. Moreover, our method is being able to predict the configurations of two binding proteins, where most of other methods predict only the binding sites. The software package is available at http://sites.google.com/site/guofeics/dobi for non-commercial use.
\end{abstract}

\section{Background}

Most of the existing efforts to identify the binding sites in protein-protein interaction are based on analyzing the differences between interface residues and non-interface residues, often through the use of machine learning or statistical methods. These methods differ in the features analyzed, that is, the sequence and structural or physical attributes. Chung et al. [1] used multiple structure alignments of the individual components in known complexes to derive structurally conserved residues. Sequence profile and accessible surface area information are combined with the conservation score to predict protein-protein binding sites by using a Support Vector Machine. Ofran et al. [2] employed neural networks to predict binding sites, using the sequence environment, the profile and the structural features as input. The random forest algorithm is

\footnotetext{
*Correspondence: cswangl@cityu.edu.hk

2 Department of Computer Science, City University of Hong Kong, 83 Tat Chee Avenue, Kowloon, Hong Kong
}

Full list of author information is available at the end of the article used to utilize these features from sequences or 3D structures for the binding site prediction [3,4]. PSIVER [5] uses sequence features for training a Nä̈ve Bayes classifier to predict binding sites. In PSIVER, conditional probabilities of each sequence feature are estimated using a kernel density estimation method.

Besides the machine learning and statistical approaches, 3D structural algorithms and other methods have also been used to identify binding sites through investigating protein surface structures. ProBiS [6] predicts binding sites by local surface structure alignment. It compares the query protein to $3 \mathrm{D}$ protein structures in a database to detect proteins with structurally similar sites on the surfaces. Burgoyne et al. [7] analyzed clefts in protein surfaces that are likely to correspond to the binding sites. They ranked them according to sequence conservation and simple measures of physical properties including hydrophobicity, desolvation, electrostatic and van der Waals potentials. Ortuso et al. [8] defined most relevant interaction areas in complexes deriving pharmacophore 
models from 3D structure information. It is based on 3D maps computed by the GRID program on structurally known molecular complexes.

ProMate [9] is based on the idea of interface and noninterface circles. A circle is first created around each residue. Then, features are extracted from these circles. Statistics are performed and histograms are created for each feature. Thereafter, the probability for each circle of a test protein to be an interface is estimated. The interface circles are clustered for each test protein to identify the binding patch.

Bradford et al. [10] proposed an approach (PPI-Pred) which uses SVM (Support Vector Machine) on surface patch features to predict binding sites. PPI-Pred generates an interacting patch and a non-interacting patch for each protein. Seven features are extracted for each patch to build an SVM model, which is then used to predict if a given test patch is an interacting patch.

In PINUP [11], an empirical scoring function is presented to predict binding sites. The function is a linear combination of energy score, interface propensity and residue conservation score. A patch is formed by a residue and its spatial neighbors within the protein subunit. PINUP takes the top 5\% scoring patches and ranks residues based on their occurrences in these patches. The top 15 ranked residues are predicted as the interface residues.

Li et al. [12] proposed another SVM approach (coreSVM). The residues of the proteins are divided into four classes: the interior residues, the core interface residues, the rim interface residues, and the non-interface residues. The core interface and rim interface residues are distinguished by the percentage of their neighboring residues which are interface residues. An SVM is built over eight features extracted from the interface residues, and used to compute the probability of whether a residue is a core interface residue.

Meta-servers have also been constructed to combine the strengths of existing approaches. The program called meta-PPISP [13] combines three individual servers, namely cons-PPISP, ProMate and PINUP; another program called metaPPI [14] combines five prediction methods, namely PPI-Pred, PINUP, PPISP, ProMate, and SPPIDER [15].

Another approach in binding site prediction is to examine the possible structural configurations, or referred to as poses, of protein subunits, that is, how the subunits may dock. Docking methods based on fast Fourier transformation (FFT) [16,17], geometric surface matching [18], as well as intermolecular energy [19-21] have been proposed. Fernández-Recio et al. [22] simulated protein docking and analyzed the interaction energy landscapes. Their method uses a global docking method based on multi-start global energy optimization of the ligand. It explores the conformational space around the whole receptor, and uses the rigid-body docking configurations to project the docking energy landscapes onto the surfaces. The low-energy regions are predicted as the binding sites.

In this paper, we propose a method which enumerates the configurations of two binding proteins (that is, the possible positions of the two subunits in a complex), and identify binding sites by evaluating the interaction between the components using the Atomic Contact Energy (ACE) function [23]. We perform rigid transformation to enumerate the configurations of two binding proteins. The enumeration is performed in conjunction with a surface identification technique for avoiding clashes between protein subunits when computing rigid transformations. The transformations which result in the minimum score according to the Atomic Contact Energy function are found; the corresponding interacting residues are reported as binding sites. Our method is implemented in a program called $\mathrm{DoBi}^{\mathrm{a}}$.

We perform experiment to compare DoBi with the existing methods using commonly used measures for assessments. The program outperforms the other methods on these measures. DoBi achieved a success rate higher than all the other methods, improving prediction quality in terms of both accuracy and coverage. In addition, it predicts the configurations of two binding proteins, as opposed to giving only the binding sites.

\section{Methods}

The main idea of our method is to enumerate "all" configurations between two proteins, where a configuration refers to the 3D coordinates representing the relative position and orientation of two protein subunits in a complex. We use the Atomic Contact Energy (ACE) function to compute the score for a configuration. The configurations with the lowest score are chosen, and the corresponding interacting residues are predicted as binding sites. We use rigid transformation to enumerate the configurations. The key techniques required here contain (1) an efficient algorithm to enumerate "all" configurations (rigid transformations) and (2) a good energy score.

\section{Atomic contact energy}

Atomic Contact Energy (ACE) is an atomic desolvation energy measure developed in [24]. It is defined over the energy of replacing a protein-atom/water contact, with a protein-atom/protein-atom contact. The ACE score takes into account 18 atom types, hence resulting in $18 \times 18$ possible atom pairs. The score for each atom pair has been determined, based on a statistical analysis of atom-pairing frequencies in known proteins. These pre-determined scores are given as log likelihood values in [24], thus allowing the summation of these values. The pre-determined 
score of effective contact energy between atom type $i$ and type $j$ is defined as

$$
T[i, j]=-\ln \frac{N_{i, j} / C_{i, j}}{\left(N_{i, 0} / C_{i, 0}\right) \times\left(N_{j, 0} / C_{j, 0}\right)}
$$

where type 0 corresponds to the solvent. The number of $i$-j contact $\left(N_{i, j}\right)$ and the number of $i-0$ contact $\left(N_{j, 0}\right)$ are estimates of the actual contact numbers of known complexes. In addition, $C_{i, j}$ and $C_{i, 0}$ are defined as the expected numbers of $i-j$ contact and $i-0$ contact.

For a given configuration, the ACE score is a summation of each of the atom pairs (one from each subunit) within threshold distance $d$, and $d=6 \AA$ is used in this paper. Denote the sets of atoms from the two subunits as $S_{1}$ and $S_{2}$, respectively, then the ACE is computed as

$$
E_{A C E}=\sum_{s \in S_{1}, t \in S_{2},|| s-t|| \leq d} T[s, t]
$$

where $\|s-t\|$ is the Euclidean distance between $s$ and $t$, and $T[s, t]$ is the pre-determined score of the atom pair $s$ and $t$.

The ACE score can be considered an estimate of the change in desolvation energy of the two proteins in going from the unbound state to the complex. A lower ACE value implies a lower (and hence more favorable) desolvation free energy.

\section{Enumeration of the configurations}

In this paper, we assume that subunits are rigid. A protein structure consists of a sequence of residues. Each residue consists of a set of atoms. We assume that the atoms in a residue are ordered as a sequence. Hence, the whole protein structure can be represented by a sequence of atoms. In the rest of this subsection, we let $A$ and $B$ denote two protein structures (subunit), and write $A=$ $\left(a_{1}, a_{2}, \ldots, b_{m}\right)$, and $B=\left(b_{1}, b_{2}, \ldots, b_{n}\right)$, where $a_{i}$, and $b_{j}$ are atoms of structure $A$ and $B$. Without loss of generality, we assume that $n \geq m$. We also assume that we know the 3D coordinates of each atom in both input proteins. We use $A[i: j]$ to denote the subsequence $\left(a_{i}, \ldots, a_{j}\right)$, and refer to a subsequence of atoms as a structural fragment.

To enumerate all the configurations, we assume $B$ is fixed, and we perform rotations and translations (referred to as rigid transformations, and simply, transformations, in the rest of the paper) on $A$. The method proposed here is modified from the algorithms for structure comparison [25].

Assume that two points $a_{i}$ and $a_{j}$ of $A$ interact with two points $b_{i^{\prime}}$ and $b_{j^{\prime}}$ of $B$, then we know that $\left\|a_{i}-b_{i^{\prime}}\right\| \leq d$ and $\left\|a_{j}-b_{j^{\prime}}\right\| \leq d$. To enumerate the configurations, we enumerate the positions for atoms $a_{i}$ and $a_{j}$ first, and for each fixed positions of $a_{i}$ and $a_{j}$, we rotate $A$ about the line formed by $a_{i}$ and $a_{j}$. Let the $d$-ball of an atom $a$ be the ball with radius $d$ centered at $a$. We discretize the $d$ ball of $b_{i^{\prime}}$ with step size $\epsilon d$, where $\epsilon$ is a small constant (and we choose $\epsilon=0.1$ for this paper). Each grid point in the $d$-ball of $b_{i^{\prime}}$ is used as a candidate position for atom $a_{i}$ for the binding. When $a_{i}$ is fixed at one of the grid points, the possible positions for $a_{j}$ form a sphere cap, where the sphere is centered at $a_{i}$ with radius $\left\|a_{i}-a_{j}\right\|$, and the cap is the portion of the spheres enclosed in the $d$-ball of $b_{j^{\prime}}$. Again, we discretize the sphere cap with step size $\epsilon d$. Each grid point on the sphere cap is a candidate position for $a_{j}$. This gives us a total of $O\left(\left(\frac{1}{\epsilon}\right)^{5}\right)$ possible positions for the pair of $a_{i}$ and $a_{j}$. After $a_{i}$ and $a_{j}$ are fixed on their respective grid points, the only degree of freedom to move $A[i, j]$ is to rotate it around the axis through $a_{i}$ and $a_{j}$. We use a $1^{\circ}$ step size; that is, we explore 360 different positions for the remaining atoms through 360 rotations. Figure 1 illustrates the steps to compute a transformation.

The method will work well if we know two interaction pairs $\left(a_{i}, b_{i^{\prime}}\right)$ and $\left(a_{j}, b_{j^{\prime}}\right)$. We can simply enumerate all the atoms pairs as the interaction pair candidate. However, there will be $O\left(n^{4}\right)$ such cases, which makes the computer program too slow in practice. This is perhaps one of the reasons that such a method has not been tried. The focus of the following subsection is to identify two pairs $\left(a_{i}, b_{i^{\prime}}\right)$ and $\left(a_{j}, b_{j^{\prime}}\right)$ which are more likely to be interaction pairs.

When enumerating "all" configurations, we also want to make sure that (1) only surface fragments can be candidate binding sites for a configuration and (2) there is no clash between the two proteins in such a configuration. Before presenting the details of the method, we define the surface atoms and clashes of two subunits first.

\section{Surface atoms}

The interface residues of two proteins are necessarily surface residues. Inspired by the work in LIGSITE ${ }^{c s c}[26,27]$, we propose a method to identify the surface atoms of a protein.

First, we build a 3D grid with step size $1 \AA$ around the protein. Then, each grid point is labeled as a protein point if it is within distance $2 \AA$ of any atom, and labeled as empty otherwise. We further subdivide the protein grid points into two types: interior or surface. A protein grid point is labeled as surface if at least one of its six neighboring grid points is empty, otherwise it is labeled as interior. With the grid points labeled, we can label the atoms. an atom is labeled as a surface atom if it is within distance $1.5 \AA$ of a surface grid point, otherwise it is labeled as an interior atom.

Figure 2 gives an example in 2D, where a protein grid point is labeled as interior if it has all four neighbors as protein points. In $3 \mathrm{D}$, a protein grid point should be labeled as interior if all of its six neighbors are labeled as protein. 


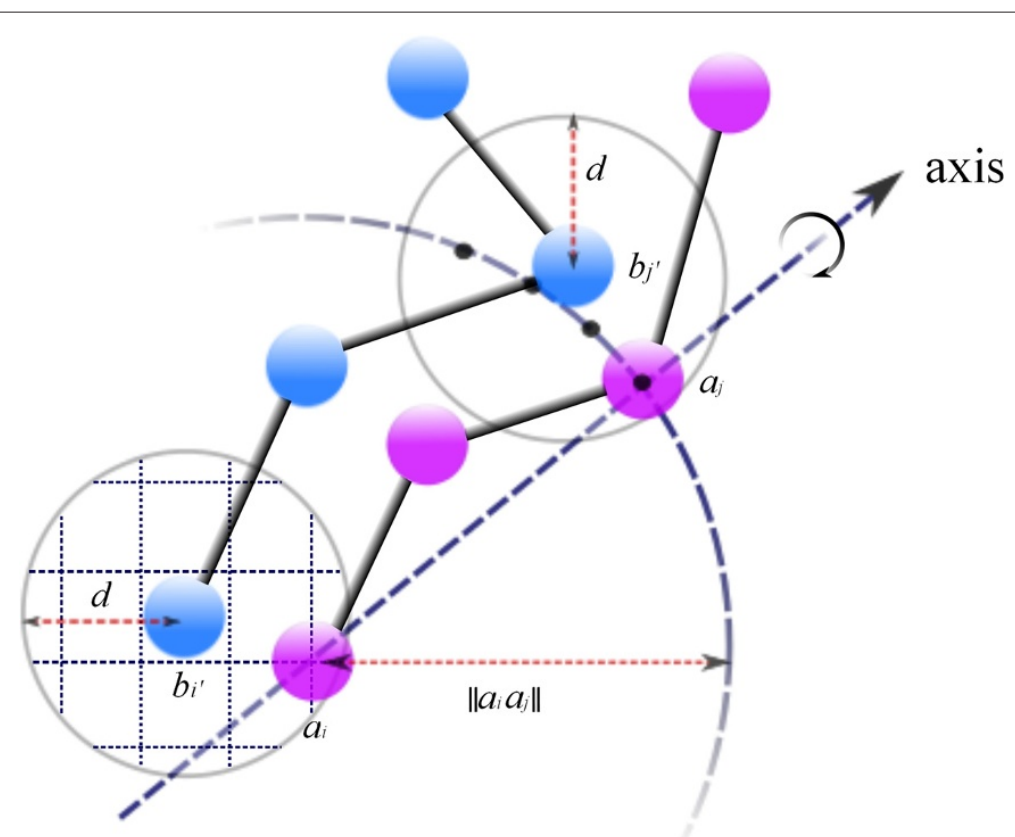

Figure 1 Steps to obtain a transformation. (1) put $a_{i}$ at one of the $O\left(\left(\frac{1}{\epsilon}\right)^{3}\right)$ grid points $d$-ball of $b_{i^{\prime}}$. (2) put $a_{j}$ at a grid point on the intersection of the sphere centered at $a_{i}$ with radius $\left|a_{i} a_{j}\right|$ and $d$-ball of $b_{j^{\prime}}$. There are at most $O\left(\left(\frac{1}{\epsilon}\right)^{2}\right)$ grid points on the intersection. (3) use $a_{i}$ and $a_{j}$ as the rotation axis.

\section{Clashes of two subunits}

A configuration cannot result in two subunits to have clashes. The following method is used to capture if a configuration resulted in clashes. Given a configuration, we build a 3D grid as in the previous subsection. For each of the structures A and B, we mark the grid points as interior, surface, or empty. We use a threshold $\theta$ to identify whether two subunits clash, by calculating the proportion of interior points for both of them. We say that the two subunits clash if they share more than $\theta \times 100 \%$ of their
A

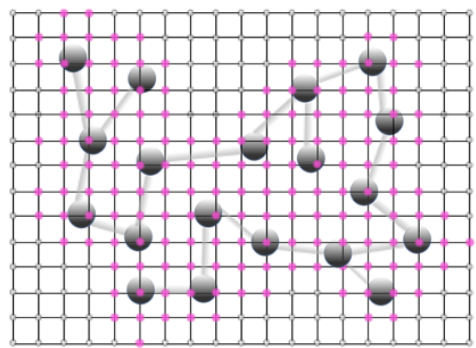

C

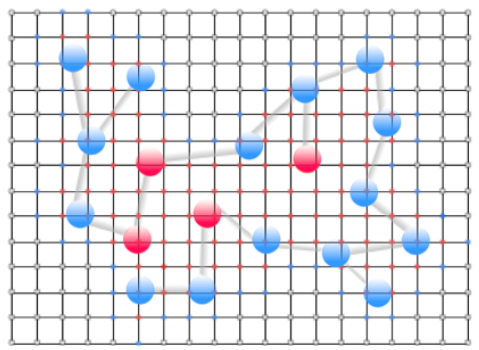

B

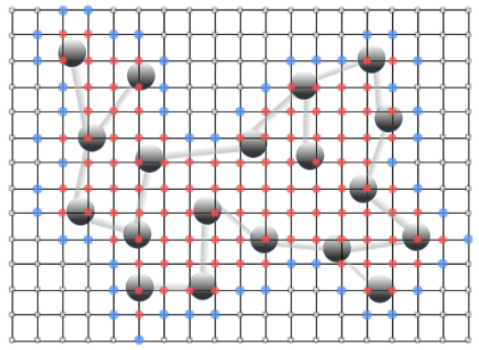

Figure $\mathbf{2}$ The surface atoms are indicated in 2D. (A) the grid is created, and grid points are labeled as either empty or protein; (B) the grid points labeled as protein are relabeled as surface or interior; (C) an atom is labeled either as surface or as interior. We use 2D as an illustration. 
interior points; that is, if $X$ is the number of interior grid points which are shared by both proteins, and $X_{A}$ and $X_{B}$ are the number of interior grid points of each subunit, respectively, then we require that $X \leq \theta \times \min \left\{X_{A}, X_{B}\right\}$ if the subunits do not clash.

\section{Finding the two interaction pairs}

In the following subsections, we present the details to explore the potential interaction pairs.

\section{Identify candidate fragment pairs}

We first select fragment pairs that are potential binding sites. As discussed in Section "Enumeration of the configurations", there are $O\left(n^{4}\right)$ possible fragment pairs $\left(a_{i}, a_{i^{\prime}}\right)$ and $\left(b_{j}, b_{j^{\prime}}\right)$ for each binding site. To reduce the computational complexity, we adopt a local alignment algorithm to accelerate this selection. This is a raw estimation and we hope that the actual binding sites are not discarded by this process.

We first use a heuristic to quickly discard fragments pairs that are unlikely to bind. The heuristic simplifies the problem, as follows: (1) every atom is within the threshold value required in the ACE computation (that is, we ignore the geometry of the structure); (2) each atom interacts with at most one atom; (3) interacting pairs follows a sequential order. That is, for any two pairs of interacted atoms $\left(a_{i}, b_{i^{\prime}}\right)$ and $\left(a_{j}, b_{j^{\prime}}\right)$, we have either $i<i^{\prime}$ and $j<j^{\prime}$, or $i^{\prime}<i$ and $j^{\prime}<j$. With these three simplifications, the standard Smith-Waterman local alignment algorithm [28] can be employed, with the ACE scores used as the penalty (negation of the score) for alignment. We use a penalty of 1 for aligning an atom to a space. Each local aligned segment gives us two fragments, where each atom in the fragment is either aligned to another atom from the partner, or aligned to nothing (i.e., aligned to space).

We present details here. For two sequences $P_{1}$ and $P_{2}$, an alignment of $P_{1}$ and $P_{2}$ can be obtained by (1) inserting spaces into the two sequences $P_{1}$ and $P_{2}$ such that the two resulting sequences with inserted spaces $P_{1}^{\prime}$ and $P_{2}^{\prime}$ have the same length and (2) overlap the two resulting sequences $P_{1}^{\prime}$ and $P_{2}^{\prime}$. The score of the alignment is the sum of the scores for all the columns, where each column has a pair of letters (including spaces) and for each pair of letters there is a pre-defined score. A subsequence $\alpha$ of $P_{1}$ and a subsequence $\beta$ of $P_{2}$ can be formed as a local aligned segment such that the score between $\alpha$ and $\beta$ is minimum. Here we want to find all (non-overlapping) pairs of subsequences with a score of at most $x$. For our purpose, we set $x=0$ throughout the paper.

Due to the simplifications, there are many false positive results, and some of the interaction pairs can be filtered. The latter issue can be handled to some extend by raising the threshold. The former issue is tackled by further refinement in the next subsection. In practice, our program outputs 70 to 120 fragment pairs as potential binding sites, which is much smaller than $O\left(n^{4}\right)$, where the number of atoms $n$ in a protein is from 500 to a few thousands.

Since a binding site is necessarily on the surface of a subunit, we filter out fragments with only very few atoms on the surface. To achieve this, we use a sliding window of length 15 to parse the aligned fragment pair. For each window, if the surface atoms are at least 2/3 (that is, ten atoms) for both fragments, the fragment pair of this window is kept for further processing and this fragment pair is extracted from the alignment. We continue this process on the un-extracted portion of the alignment. If the window does not contain sufficient surface atoms, we continue at the next window. Our choice of $2 / 3$ comes from observations with a docking decoy set from the Dockground [29], where $94 \%$ of the binding sites have more than $2 / 3$ of surface atoms.

\section{Identify configurations of fragment pairs}

From the fragment pairs obtained in the previous step, a second step is used to further filter out fragment pairs of ACE scores below a threshold. Given two structural fragments $A[i, j]=\left(a_{i}, \ldots, a_{j}\right)$, and $B\left[i^{\prime}, j^{\prime}\right]=\left(b_{i^{\prime}}, \ldots, b_{j^{\prime}}\right)$, we assume that $a_{i}$ interacts with $b_{i^{\prime}}$, and $a_{j}$ interacts with $b_{j^{\prime}}$. Using the enumeration method described earlier, we enumerate different configurations for $A$ and $B$ and compute the corresponding ACE score for the atom sets $A[i, j]$ and $B\left[i^{\prime}, j^{\prime}\right]$. We do not consider any configuration which causes $A$ and $B$ to clash. In this step, a pair of structural fragment which does not give any configuration with an ACE score below a specified threshold is discarded. In this paper, we define the threshold value as 400 , since the ACE scores of actual interface in the docking decoy set from Dockground are all less than 400. After this step, it is unlikely for two protein structures which cannot be bound to have an unfiltered fragment pair.

\section{Identify the configuration for the two subunits}

In the third step, for each pair of protein structures with at least one remaining fragment pair, we enumerate all the potential configurations for the structures. We want to use the begin and end atoms of the identified fragments for our choice of $\left(a_{i}, b_{i^{\prime}}\right)$ and $\left(a_{j}, b_{j^{\prime}}\right)$ in the enumeration, since these are the atoms that are likely to be interacting. Assuming that there are $k$ fragment pairs from the same two proteins left after the filtration of the second step, we will have a maximum of $2 k$ distinct atom pairs to choose. Thus, there is a total of at most $\left(\begin{array}{c}2 k \\ 2\end{array}\right)$ combinations to consider for the choice of $\left(a_{i}, b_{i^{\prime}}\right)$ and $\left(a_{j}, b_{j^{\prime}}\right)$.

When the best configuration is obtained, two residues, one from each subunit, are reported as the interface residues if they can be connected with a pair of atoms within distance $4.5 \AA$. In our search for the best 
Table 1 Details of DoBi on the training set

\begin{tabular}{|c|c|c|c|c|c|c|c|c|c|c|c|}
\hline Complex & $\mathrm{F}_{r}^{\mathrm{a}}$ & $\mathrm{F}_{l}^{\mathrm{b}}$ & Complex & $F_{r}$ & $F_{l}$ & Complex & $F_{r}$ & $F_{I}$ & Complex & $\mathrm{F}_{r}$ & $F_{I}$ \\
\hline $1 \mathrm{a} 2 \mathrm{x}(\mathrm{A}: \mathrm{B})$ & 45.8 & 73.7 & 1jtd(A:B) & 59.5 & 51.2 & $1 \mathrm{r} 1 \mathrm{k}(\mathrm{A}: \mathrm{D})$ & 24.5 & 12.0 & $1 z 3 g(H: A)$ & 77.4 & 85.7 \\
\hline $1 a 2 y(A: C)$ & 77.8 & 60.9 & 1jtp(A:L) & 62.9 & 70.0 & $1 \operatorname{rzr}(\mathrm{C}: T)$ & 60.5 & 70.3 & $1 z 5 s(A: B)$ & 52.2 & 66.7 \\
\hline 1 aip(A:C) & 64.4 & 59.1 & 1jwm(A:D) & 61.5 & 58.8 & $1 \mathrm{~s} 3 \mathrm{~s}(\mathrm{~F}: \mathrm{G})$ & 61.0 & 66.7 & 1z92(A:B) & 27.0 & 46.2 \\
\hline 1ava(A:C) & 74.2 & 60.3 & 1 k93(A:D) & 37.6 & 33.7 & $1 \operatorname{sgp}(\mathrm{E}: \mathrm{l})$ & 53.7 & 58.3 & $1 z \mid h(A: B)$ & 62.5 & 64.0 \\
\hline $1 \mathrm{bnd}(\mathrm{A}: \mathrm{B})$ & 53.1 & 57.1 & $1 \mathrm{kkm}(\mathrm{A}: \mathrm{l})$ & 51.9 & 58.5 & 1shw(B:A) & 72.7 & 76.9 & $1 z m 2(A: B)$ & 46.9 & 52.5 \\
\hline 1 bzq(A:L) & 64.9 & 75.0 & $1 \mathrm{kps}(\mathrm{A}: \mathrm{B})$ & 68.8 & 62.5 & 1sqo(B:A) & 50.0 & 57.1 & 2a19(B:A) & 76.9 & 72.2 \\
\hline $1 c 9 p(A: B)$ & 61.8 & 54.5 & 1 ktk(E:A) & 30.8 & 61.5 & 1sq2(L:N) & 73.7 & 78.8 & $2 \mathrm{a} 41(\mathrm{~A}: \mathrm{C})$ & 76.4 & 90.2 \\
\hline $1 \mathrm{cgj}(\mathrm{E}: \mathrm{l})$ & 65.3 & 63.4 & 1 ku6(A:B) & 63.0 & 83.3 & $1 \operatorname{ta} 3(B: A)$ & 34.6 & 53.7 & 2a42(A:B) & 79.1 & 70.2 \\
\hline $1 \mathrm{cxz}(\mathrm{A}: \mathrm{B})$ & 54.5 & 60.0 & $114 d(A: B)$ & 81.0 & 66.7 & 1te1(A:B) & 78.3 & 83.6 & $2 \mathrm{a} 5 \mathrm{~d}(\mathrm{~B}: \mathrm{A})$ & 73.3 & 84.4 \\
\hline $1 \mathrm{~d} 4 \times(\mathrm{A}: \mathrm{G})$ & 59.6 & 72.7 & $1 \mathrm{~m} 27(\mathrm{~A}: \mathrm{C})$ & 76.2 & 78.3 & 1tk5(A:B) & 65.6 & 47.2 & 2auh(A:B) & 60.0 & 77.3 \\
\hline $1 \mathrm{dfg}(\mathrm{A}: \mathrm{C})$ & 45.0 & 58.3 & 1 ma9(A:B) & 12.9 & 60.3 & 1 tu3(A:F) & 82.8 & 76.9 & 2b12(A:B) & 71.0 & 57.1 \\
\hline $1 \mathrm{dhk}(\mathrm{A}: \mathrm{B})$ & 10.8 & 57.6 & $1 \mathrm{mbx}(\mathrm{A}: \mathrm{C})$ & 48.9 & 64.7 & $1 \mathrm{u} 0 \mathrm{n}(\mathrm{A}: \mathrm{D})$ & 18.2 & 19.5 & $2 b 3 t(B: A)$ & 68.9 & 59.5 \\
\hline $1 \mathrm{dkf}(\mathrm{B}: \mathrm{A})$ & 47.8 & 68.2 & $1 \mathrm{mr} 1(\mathrm{~A}: \mathrm{D})$ & 83.7 & 77.4 & $1 \mathrm{u} 0 \mathrm{~s}(\mathrm{Y}: \mathrm{A})$ & 89.5 & 90.9 & $2 b 5 i(B: A)$ & 78.8 & 62.5 \\
\hline $1 \mathrm{dp} 5(\mathrm{~A}: \mathrm{B})$ & 74.2 & 86.8 & $1 \mathrm{mzw}(\mathrm{A}: \mathrm{B})$ & 55.2 & 72.7 & 1 u7e(A:B) & 26.9 & 62.5 & $2 b h 1(A: X)$ & 60.9 & 57.9 \\
\hline 1eai(B:D) & 52.2 & 70.6 & 1nby(A:C) & 50.0 & 58.3 & 1 uex(A:C) & 27.3 & 50.0 & $2 b k h(A: B)$ & 74.3 & 67.9 \\
\hline 1 efu(C:D) & 57.1 & 70.3 & 1ncb(L:N) & 48.6 & 30.8 & $1 \mathrm{ujw}(\mathrm{A}: \mathrm{B})$ & 36.1 & 82.8 & 2bkk(A:B) & 74.3 & 52.6 \\
\hline $1 f 5 q(A: B)$ & 58.2 & 63.0 & $1 \mathrm{nmu}(\mathrm{A}: \mathrm{B})$ & 43.9 & 51.6 & $1 \mathrm{ul}(\mathrm{X}: \mathrm{A})$ & 52.6 & 51.4 & 2bnq(D:A) & 51.9 & 34.5 \\
\hline $1 \mathrm{f} 6 \mathrm{a}(\mathrm{B}: \mathrm{A})$ & 28.6 & 47.6 & 1 npe(A:B) & 43.1 & 68.1 & 1 uuz(A:D) & 58.8 & 57.9 & $2 \mathrm{c} 1 \mathrm{~m}(\mathrm{~A}: \mathrm{B})$ & 40.4 & 66.7 \\
\hline $1 f 7 z(A: I)$ & 72.7 & 89.7 & 1nu9(A:C) & 56.7 & 56.8 & $1 \mathrm{uzx}(\mathrm{A}: \mathrm{B})$ & 71.0 & 68.7 & $2 c 5 d(A: C)$ & 54.2 & 69.4 \\
\hline $1 \mathrm{ffg}(\mathrm{A}: \mathrm{B})$ & 73.3 & 62.1 & $10 i u(A: B)$ & 70.8 & 76.2 & $1 v 5 i(A: B)$ & 3.8 & 87.2 & 2gy7(B:A) & 63.2 & 73.2 \\
\hline $1 \mathrm{fm} 9(\mathrm{D}: \mathrm{A})$ & 82.6 & 89.4 & $10 m w(A: B)$ & 75.8 & 63.4 & $1 \vee 7 p(A: C)$ & 50.0 & 41.4 & 2hdi(A:B) & 9.1 & 57.1 \\
\hline 1 fns $(L: A)$ & 50.0 & 28.6 & 1 p3q(R:V) & 66.7 & 80.0 & 1 w98(A:B) & 50.7 & 62.3 & $2 \operatorname{iw} 5(A: B)$ & 66.1 & 72.5 \\
\hline 1g20(A:E) & 45.8 & 40.8 & $1 p 7 q(A: D)$ & 63.6 & 61.5 & $1 w p x(A: B)$ & 58.3 & 55.2 & 2j0m(A:B) & 81.3 & 64.3 \\
\hline 1g9m(G:L) & 38.1 & 28.6 & 1p9m(C:B) & 85.7 & 70.6 & 1 wr6(A:E) & 89.7 & 93.3 & 2jbo(B:A) & 66.7 & 63.2 \\
\hline $1 \mathrm{hod}(\mathrm{A}: \mathrm{C})$ & 16.7 & 30.0 & $1 \mathrm{pkq}(\mathrm{A}: \mathrm{E})$ & 27.3 & 9.1 & $1 \mathrm{wrd}(\mathrm{A}: \mathrm{B})$ & 56.2 & 69.2 & $20 m z(A: B)$ & 60.2 & 71.0 \\
\hline 1h59(A:B) & 91.7 & 81.5 & 1 ppf(E:I) & 85.1 & 83.9 & $1 \times 86(A: B)$ & 52.6 & 60.4 & $2 \mathrm{p} 8 \mathrm{w}(\mathrm{T}: \mathrm{S})$ & 56.6 & 90.3 \\
\hline 1i8|(A:C) & 83.9 & 71.4 & $1 \operatorname{qav}(B: A)$ & 81.3 & 78.0 & $1 x d t(T: R)$ & 48.4 & 90.2 & $2 p a v(A: P)$ & 72.0 & 73.1 \\
\hline $1 \operatorname{iar}(B: A)$ & 76.5 & 51.6 & 1qbk(B:C) & 41.9 & 38.6 & $1 \times \times 9(C: A)$ & 54.5 & 40.0 & 3bp5(B:A) & 70.0 & 72.0 \\
\hline 1j|4(A:D) & 40.0 & 44.4 & $1 \mathrm{qo0}(\mathrm{B}: \mathrm{A})$ & 31.6 & 33.3 & 1 yi5(A:F) & 83.9 & 76.5 & 3ygs(C:P) & 64.5 & 58.1 \\
\hline
\end{tabular}

${ }^{\mathrm{a}} \mathrm{F}_{r}(\%)$ is the $\mathrm{F}$-score of our method on the receptor proteins.

${ }^{\mathrm{b}} \mathrm{F}_{/}(\%)$ is the $\mathrm{F}$-score of our method on the ligand proteins.

Table 2 Comparison of DoBi and Fernández-Recio et al.'s method

\begin{tabular}{|c|c|c|c|c|c|c|c|c|c|c|c|c|}
\hline & \multicolumn{6}{|c|}{ DoBi } & \multicolumn{6}{|c|}{ Fernández-Recio et al.'s } \\
\hline & Suc $^{\mathrm{a}}$ & $A c c^{b}$ & $\operatorname{Cov}^{\mathrm{c}}$ & $F^{f}$ & $M^{\mathrm{d}}$ & $V^{\mathrm{e}}$ & Suc & Acc & Cov & $F$ & $M$ & $v$ \\
\hline Overall & 39.6 & 44.3 & 70.5 & 0.54 & 37.5 & 29.0 & 37.2 & 39.3 & 72.7 & 0.51 & 46.3 & 40.0 \\
\hline
\end{tabular}

\footnotetext{
${ }^{a}$ Suc (\%) is the success rate of the corresponding method on the data set.

${ }^{\mathrm{b}} \mathrm{Acc}(\%)$ is the average accuracy of the corresponding method on the data set.

${ }^{c} \mathrm{Cov}(\%)$ is the average coverage of the corresponding method on the data set.

${ }^{\mathrm{d}} M$ is the average of the sizes predicted by the corresponding method on the data set.

${ }^{\mathrm{e}} V$ is the standard deviation of the sizes predicted by the corresponding method on the data set

${ }^{f} \mathrm{~F}$ is the F-score of the corresponding method on the data set.
} 
Table 3 Detailed Results of DoBi and Fernández-Recio et al.'s method

\begin{tabular}{|c|c|c|c|c|c|c|c|c|c|c|c|c|}
\hline \multirow{3}{*}{ Complex } & \multicolumn{6}{|c|}{ Receptor } & \multicolumn{6}{|c|}{ Ligand } \\
\hline & \multirow[b]{2}{*}{ PDB $^{\mathrm{a}}$} & \multirow[b]{2}{*}{$\operatorname{lnt}_{\mathrm{n}}{ }^{\mathrm{b}}$} & \multicolumn{2}{|c|}{ DoBi } & \multicolumn{2}{|c|}{ Fernández-Recio ${ }^{\mathrm{e}}$} & \multirow[b]{2}{*}{ PDB } & \multirow[b]{2}{*}{$\operatorname{lnt} t_{\mathrm{n}}$} & \multicolumn{2}{|c|}{ DoBi } & \multicolumn{2}{|c|}{ Fernández-Recio } \\
\hline & & & $A c C^{\mathrm{C}}$ & $\operatorname{Cov}^{d}$ & $A c c$ & $\operatorname{Cov}$ & & & $A c c$ & Cov & Acc & Cov \\
\hline $1 \mathrm{caO}(\mathrm{B}: \mathrm{D})$ & 5 cha & 24 & 46.2 & 50.0 & 50.6 & 81.0 & 1 aap & 14 & 26.1 & 42.9 & 35.6 & 57.0 \\
\hline $1 \mathrm{cbw}(\mathrm{B}: \mathrm{D})$ & 5 cha & 26 & 58.6 & 65.4 & 65.7 & 92.0 & $1 \mathrm{bpi}$ & 14 & 77.8 & 100 & 33.7 & 64.0 \\
\hline $1 \mathrm{acb}(\mathrm{E}: \mathrm{I})$ & 5 cha & 24 & 14.5 & 66.7 & 55.0 & 77.0 & legl & 13 & 20.4 & 84.6 & 21.6 & 41.0 \\
\hline 1cho(F:I) & 5 cha & 25 & 36.9 & 96.0 & 63.6 & 89.0 & 1omu & 13 & 35.3 & 92.3 & 48.1 & 77.0 \\
\hline 1cgi(E:I) & 1chg & 24 & 26.3 & 45.5 & 70.8 & 92.0 & $1 \mathrm{hpt}$ & 19 & 48.5 & 84.2 & 58.3 & 70.0 \\
\hline 2kai(A:I) & $2 p k a$ & 33 & 53.8 & 58.3 & 41.5 & 54.0 & $1 \mathrm{bpi}$ & 19 & 68.8 & 84.6 & 35.9 & 79.0 \\
\hline 2sni(E:I) & $2 s t 1$ & 28 & 61.1 & 78.6 & 35.8 & 93.0 & $2 \mathrm{ci} 2$ & 15 & 70.6 & 80.0 & 37.9 & 53.0 \\
\hline $2 \operatorname{sic}(E: I)$ & $2 s t 1$ & 30 & 73.5 & 83.3 & 29.6 & 83.0 & $3 s s i$ & 12 & 62.5 & 83.3 & 18.4 & 46.0 \\
\hline 1cse(E:I) & $1 \mathrm{sbc}$ & 30 & 42.6 & 96.7 & 33.1 & 96.0 & 1egl & 12 & 26.3 & 83.3 & 22.8 & 41.0 \\
\hline $2 \operatorname{tec}(\mathrm{E}: I)$ & 1thm & 28 & 38.0 & 67.9 & 34.2 & 82.0 & 1egl & 13 & 31.0 & 69.2 & 30.0 & 45.0 \\
\hline $1 \operatorname{taw}(A: B)$ & $5 p t p$ & 26 & 42.1 & 30.8 & 51.9 & 83.0 & 1aap & 13 & 47.1 & 61.5 & 34.4 & 62.0 \\
\hline $2 p t c(E: I)$ & $5 p t p$ & 24 & 33.3 & 50.0 & 52.4 & 89.0 & $1 \mathrm{bpi}$ & 14 & 56.5 & 92.9 & 18.0 & 36.0 \\
\hline $3 \operatorname{tgi}(\mathrm{E}: \mathrm{l})$ & lane & 25 & 51.9 & 56.0 & 16.1 & 29.0 & $1 \mathrm{bpi}$ & 14 & 58.8 & 71.4 & 30.5 & 64.0 \\
\hline $1 \mathrm{brc}(\mathrm{E}: \mathrm{I})$ & $1 \mathrm{bra}$ & 24 & 30.0 & 25.0 & 44.4 & 80.0 & 1aap & 11 & 62.5 & 90.9 & 36.5 & 62.0 \\
\hline $1 \mathrm{fss}(\mathrm{A}: \mathrm{B})$ & 2ace & 25 & 32.7 & 64.0 & 23.8 & 100 & $1 \mathrm{fsC}$ & 19 & 65.4 & 89.5 & 69.2 & 83.0 \\
\hline $1 \mathrm{bvn}(\mathrm{P}: \mathrm{T})$ & 1 pif & 31 & 29.2 & 22.6 & 45.0 & 90.0 & 2ait & 20 & 42.1 & 80.0 & 61.4 & 86.0 \\
\hline $1 \mathrm{bgs}(\mathrm{B}: \mathrm{F})$ & $1 a 2 p$ & 18 & 23.1 & 66.7 & 73.1 & 95.0 & $1 \mathrm{a} 19$ & 16 & 34.1 & 93.8 & 72.3 & 94.0 \\
\hline 1ay7(A:B) & 1rge & 15 & 81.3 & 86.7 & 71.4 & 100 & $1 \mathrm{a} 19$ & 15 & 84.6 & 73.3 & 52.2 & 94.0 \\
\hline 1ugh(E:I) & 1akz & 24 & 63.6 & 87.5 & 44.1 & 97.0 & 2ugi & 25 & 57.1 & 64.0 & 83.3 & 75.0 \\
\hline $2 p c b(A: B)$ & $1 c c p$ & 10 & 23.5 & 40.0 & 24.2 & 92.0 & $1 \mathrm{hrc}$ & 9 & 22.2 & 44.4 & 29.2 & 73.0 \\
\hline $2 p c f(B: A)$ & $1 \mathrm{ctm}$ & 21 & 57.7 & 71.4 & 57.5 & 92.0 & 1ag6 & 24 & 56.7 & 70.8 & 66.4 & 73.0 \\
\hline $1 \mathrm{mlc}(\mathrm{B}: \mathrm{E})$ & $1 \mathrm{mlb}$ & 14 & 65.0 & 92.9 & 31.3 & 100 & 1 lza & 10 & 43.5 & 100 & 9.1 & 29.0 \\
\hline $1 \mathrm{vfb}(\mathrm{A}: \mathrm{C})$ & $1 \mathrm{vfa}$ & 8 & 44.4 & 100 & 52.6 & 100 & 1 lza & 8 & 43.8 & 87.5 & 26.8 & 83.0 \\
\hline 1ewy(A:C) & 1que & 15 & 20.8 & 26.3 & 52.6 & 100 & $1 f x a$ & 15 & 37.5 & 52.9 & 56.7 & 68.0 \\
\hline 1eer(B:A) & 1ern & 23 & 13.8 & 65.2 & 35.0 & 91.0 & 1 buy & 22 & 21.9 & 95.5 & 53.6 & 75.0 \\
\hline $1 \mathrm{kkl}(\mathrm{A}: \mathrm{H})$ & 1jb1 & 13 & 31.3 & 76.9 & 3.5 & 11.0 & $1 \mathrm{sph}$ & 12 & 32.4 & 100 & 67.5 & 81.0 \\
\hline 1 ken(A:C) & 2 viu & 56 & 92.6 & 44.6 & 30.3 & 97.0 & 1 ken & 64 & 71.7 & 51.6 & 29.4 & 100 \\
\hline $1 \mathrm{kxv}(\mathrm{A}: \mathrm{C})$ & 1 pif & 19 & 15.0 & 63.2 & 3.7 & 10.0 & $1 \mathrm{kxv}$ & 21 & 27.0 & 81.0 & 43.7 & 83.0 \\
\hline $1 \mathrm{kxt}(\mathrm{A}: \mathrm{B})$ & 1 pif & 17 & 17.9 & 41.2 & 14.1 & 55.0 & $1 \mathrm{kxt}$ & 20 & 30.8 & 40.0 & 53.3 & 96.0 \\
\hline $1 \mathrm{kxq}(\mathrm{A}: \mathrm{H})$ & 1 pif & 30 & 42.5 & 56.7 & 52.6 & 100 & $1 \mathrm{kxq}$ & 25 & 54.5 & 72.0 & 56.5 & 96.0 \\
\hline $110 x(A: B)$ & $1 \mathrm{bec}$ & 19 & 42.9 & 40.0 & 0 & 0 & 1b1z & 17 & 27.8 & 41.7 & 16.1 & 100 \\
\hline $1 \operatorname{avw}(A: B)$ & $2 \mathrm{ptn}$ & 31 & 31.3 & 48.4 & 58.8 & 100 & 1 ba7 & 15 & 44.1 & 100 & 36.2 & 94.0 \\
\hline $1 \mathrm{dfj}(\mathrm{l}: \mathrm{E})$ & $2 b n h$ & 33 & 52.9 & 54.5 & 49.4 & 89.0 & 7rsa & 29 & 47.1 & 55.2 & 66.7 & 80.0 \\
\hline $1 \operatorname{tgs}(Z: I)$ & $2 p t n$ & 30 & 30.4 & 70.0 & 62.0 & 93.0 & $1 \mathrm{hpt}$ & 18 & 43.8 & 77.8 & 68.3 & 82.0 \\
\hline $1 a h w(A: B)$ & $1 \mathrm{fgn}$ & 43 & 23.0 & 39.5 & 15.6 & 89.0 & 1 boy & 45 & 28.3 & 62.2 & 0 & 0 \\
\hline $1 \mathrm{dqj}(\mathrm{A}: \mathrm{C})$ & 1dqq & 11 & 50.0 & 81.8 & 20.0 & 100 & $31 z t$ & 11 & 50.0 & 81.8 & 14.4 & 39.0 \\
\hline 1 wej(H:F) & $1 \mathrm{qbl}$ & 7 & 38.9 & 100 & 24.4 & 100 & $1 \mathrm{hrc}$ & 8 & 40.0 & 100 & 18.3 & 44.0 \\
\hline $1 \operatorname{avz}(B: C)$ & lavv & 16 & 58.8 & 62.5 & 16.2 & 42.0 & $1 \mathrm{shf}$ & 13 & 42.3 & 84.6 & 54.1 & 92.0 \\
\hline $1 \mathrm{wq} 1$ (G:R) & 1 wer & 33 & 70.6 & 72.7 & 11.4 & 33.0 & $5 p 21$ & 26 & 77.8 & 80.8 & 40.8 & 53.0 \\
\hline $2 m t a(L: A)$ & $2 \mathrm{bbk}$ & 13 & 57.9 & 84.6 & 30.0 & 93.0 & 1 aan & 11 & 64.7 & 100 & 58.8 & 100 \\
\hline
\end{tabular}


Table 3 Detailed Results of DoBi and Fernández-Recio et al.'s method (Continued)

\begin{tabular}{|c|c|c|c|c|c|c|c|c|c|c|c|c|}
\hline $1 \mathrm{bth}(\mathrm{H}: \mathrm{P})$ & $2 \mathrm{hnt}$ & 30 & 15.2 & 16.7 & 27.7 & 61.0 & $6 p t i$ & 17 & 94.1 & 94.1 & 32.5 & 39.0 \\
\hline 1 fin $(A: B)$ & $1 \mathrm{hcl}$ & 46 & 35.5 & 47.8 & 28.3 & 68.0 & $1 \mathrm{vin}$ & 35 & 32.8 & 60.0 & 66.7 & 100 \\
\hline $1 \mathrm{fq} 1(\mathrm{~B}: \mathrm{A})$ & $1 b 39$ & 16 & 63.2 & 75.0 & 8.2 & 32.0 & $1 \mathrm{fpz}$ & 16 & 63.2 & 75.0 & 0 & 0 \\
\hline
\end{tabular}

a PDB is the unbound structure of the receptor or ligand in the complex.

$\mathrm{b} / n t_{\mathrm{n}}$ is the number of residues on the actual interface in the complex.

${ }^{\mathrm{C}} \mathrm{Acc}(\%)$ is the accuracy of the corresponding method on the data set.

${ }^{d} \operatorname{Cov}(\%)$ is the coverage of the corresponding method on the data set.

eThe values for this method are from literature [22].

configuration, we also require the configurations to be free from clashes.

\section{Results and discussion}

Three commonly used measures are utilized to assess the performance of DoBi. Accuracy and Coverage are two common measures to assess the quality of the binding sites adopted by a method [11]. The accuracy of the predicted interface is the fraction of correctly predicted residues over the total number of predicted interface residues; the coverage of the predicted interface is the fraction of correctly predicted interface residues over the total number of actual interface residues. F-score $(F=$ $\left.2 \times \frac{\text { Accuracy } \times \text { Coverage }}{\text { Accuracy+Coverage }}\right)$ is a weighted average of the accuracy and coverage, where an F-score reaches its best score at 1 and worst score at 0 . Another common measure is success rate, which is defined in [9]. A reported result is claimed as a success if at least half of the predicted residues are actual interface residues; that is, the accuracy is no less than $50 \%$. The success rate is the fraction of successful predicted cases in the total number of predicted proteins.

A protein complex may contain several subunits, and multiple binding sites. Each binding site in a protein complex consists of a pair of subunits. Two residues in a pair of subunits are called interface residues if any two atoms, one from each residue, interact. By interact, we mean the distance between the two atoms is less than the sum of the van der Waals radius of the two atoms plus $1 \AA$. The number of residues on interface is referred to as the interface size.

\section{Training set}

We use the unbound protein structures from Dockground [29] as the training set to calculate the parameters of DoBi. The docking decoys from Dockground were generated by GRAMM-X scan. The GRAMM-X docking scan was used to generate 102 unbound-unbound complexes and 131 unbound-bound complexes. By excluding the proteins used in the comparison, 36 unbound-unbound complexes and 80 unbound-bound complexes can be used to calculate the value of the threshold $\theta$. When we set $\theta=0.17$, the overall F-score of DoBi on the training set is $60.5 \%$, which is the best score that DoBi achieves under different threshold values. The details on the training set are shown in Table 1.

\section{Comparison to the existing methods}

We divide our comparisons into four separate groups, where in each group we compare a different set of methods. The reason that we cannot compare all the methods with the same data set is due to the unavailability of some methods, in which case the only comparison possible is with the results in the respective publications.

\section{Comparison to Fernández-Recio et al.'s method}

DoBi is compared to the method introduced by Fernández-Recio et al. in [22], using the test data therein, which consists of 43 complexes. The results are reported in Table 2. The overall accuracy and coverage for DoBi are $44.3 \%$ and $70.5 \%$. Fernández-Recio et al.'s method achieved the overall accuracy and coverage of $39.3 \%$ and $72.7 \%$, respectively. The success rate for DoBi is $39.6 \%$, improving over the success rate of $37.2 \%$ reported by Fernández-Recio et al. The F-score is 0.54 for DoBi, and 0.51 for Fernández-Recio et al.'s method.

The average predicted sizes for DoBi and FernándezRecio et al.'s method are 37.5 residues and 46.3 residues respectively, while the average actual size is 21.1 residues. The standard deviation of the sizes predicted by DoBi is 29.0, while that of the sizes predicted by Fernández-Recio et al.'s method is 40.0 .

Table 3 displays the detailed results for all unbound structures of 43 complexes. Each row corresponds to a pair of proteins. We can observe from the table that the binding sites are identified accurately for the complexes 2sni(E:I), 2sic(E:I), 1ay7(A:B) and 1wq1(G:R).

\section{Comparison to metaPPI, meta-PPISP and PPI-Pred}

In this group of our comparisons, the test set in [14] is used. It consists of 41 complexes from the benchmark v2.0 [30] and 27 targets from the CAPRI experiment[31]. The 41 complexes are divided into two categories, enzymeinhibitor (EI) and others. We compare our method to metaPPI, meta-PPISP and PPI-Pred with this group of data. The overall accuracy and coverage of each prediction method are shown in Table 4. DoBi has an F-score of 0.55, where in contrast, metaPPI, meta-PPISP and PPI-Pred 
Table 4 Comparisons of DoBi, metaPPI, meta-PPISP and PPI-Pred

\begin{tabular}{|c|c|c|c|c|c|c|c|c|c|c|c|c|c|c|c|c|c|c|c|c|c|c|c|c|}
\hline \multirow[b]{2}{*}{ Type } & \multicolumn{6}{|c|}{ DoBi } & \multicolumn{6}{|c|}{ metaPPI } & \multicolumn{6}{|c|}{ meta-PPISP } & \multicolumn{6}{|c|}{ PPI-Pred } \\
\hline & $\mathrm{Suc}^{\mathrm{a}}$ & $\mathrm{Acc}^{\mathrm{b}}$ & $\operatorname{Cov}^{c}$ & $\mathrm{~F}^{\mathrm{g}}$ & $M^{\mathrm{e}}$ & $V^{f}$ & Suc & Acc & Cov & $\mathrm{F}$ & $M$ & $v$ & Suc & Acc & Cov & $F$ & $M$ & $v$ & Suc & Acc & Cov & $F$ & $M$ & $V$ \\
\hline$E-\left.\right|^{d}$ & 67.6 & 56.7 & 61.9 & 0.59 & 23.0 & 7.6 & 70.5 & 61.1 & 36.5 & 0.45 & 12.9 & 10.4 & 55.8 & 56.4 & 54.7 & 0.55 & 24.1 & 13.5 & 47.1 & 39.5 & 37.9 & 0.38 & 23.7 & 15.1 \\
\hline others & 47.9 & 46.4 & 63.3 & 0.53 & 29.5 & 19.8 & 43.8 & 40.7 & 22.2 & 0.28 & 8.0 & 10.1 & 35.6 & 38.5 & 25.7 & 0.30 & 11.8 & 12.6 & 22.9 & 29.3 & 31.3 & 0.30 & 19.0 & 14.7 \\
\hline CAPRI & 50.0 & 48.9 & 55.8 & 0.52 & 25.7 & 12.3 & 50.0 & 46.7 & 24.3 & 0.32 & 15.7 & 12.8 & 26.0 & 27.9 & 30.8 & 0.29 & 19.6 & 13.8 & 28.6 & 25.7 & 29.5 & 0.27 & 28.2 & 19.2 \\
\hline Overall & 53.7 & 50.0 & 60.0 & 0.55 & 26.4 & 13.8 & 52.9 & 48.2 & 26.6 & 0.35 & 12.3 & 11.2 & 36.8 & 38.8 & 35.0 & 0.43 & 18.0 & 13.3 & 31.2 & 30.4 & 32.2 & 0.32 & 23.8 & 16.6 \\
\hline
\end{tabular}

a Suc (\%) is the success rate of the corresponding method on the data set.

${ }^{\mathrm{b}} \mathrm{Acc}(\%)$ is the average accuracy of the corresponding method on the data set.

${ }^{\mathrm{C}} \mathrm{Cov}(\%)$ is the average coverage of the corresponding method on the data set.

${ }^{d} E-l$ is the type of enzyme-inhibitor.

${ }^{\mathrm{e}} M$ is the average of the sizes predicted by the corresponding method on the data set.

${ }^{f} V$ is the standard deviation of the sizes predicted by the corresponding method on the data set.

${ }^{9} \mathrm{~F}$ is the $\mathrm{F}$-score of the corresponding method on the data set. 
Table 5 Detailed Results of DoBi, metaPPI, meta-PPISP and PPI-Pred on 41 complexes

\begin{tabular}{|c|c|c|c|c|c|c|c|c|c|c|c|c|c|c|c|c|c|c|c|c|}
\hline \multirow[t]{3}{*}{ Complex } & \multicolumn{10}{|c|}{ Protein 1} & \multicolumn{10}{|c|}{ Protein 2} \\
\hline & \multirow[t]{2}{*}{$\mathrm{PDB}^{\mathrm{a}}$} & \multirow[t]{2}{*}{$I n t_{n}{ }^{b}$} & \multicolumn{2}{|c|}{ DoBi } & \multicolumn{2}{|c|}{ metaPPI ${ }^{f}$} & \multicolumn{2}{|c|}{ meta-PPISPf } & \multicolumn{2}{|c|}{ PPI-Pred $^{9}$} & \multirow[t]{2}{*}{$\overline{P D B}$} & \multirow[t]{2}{*}{$\operatorname{lnt} t_{\mathrm{n}}$} & \multicolumn{2}{|c|}{ DoBi } & \multicolumn{2}{|c|}{ metaPPI } & \multicolumn{2}{|c|}{ meta-PPISP } & \multicolumn{2}{|c|}{ PPI-Pred } \\
\hline & & & $A c c^{c}$ & $\operatorname{Cov}^{\mathrm{d}}$ & $A c c$ & $\operatorname{Cov}$ & $A c c$ & Cov & Acc & $\operatorname{Cov}$ & & & Acc & $\operatorname{Cov}$ & $A c c$ & $\operatorname{Cov}$ & Acc & $\operatorname{Cov}$ & $A c c$ & $\mathrm{Cov}$ \\
\hline \multicolumn{21}{|l|}{$\overline{E-l^{e}}$} \\
\hline $1 \mathrm{acb}(\mathrm{E}: \mathrm{l})^{\mathrm{h}}$ & $2 \mathrm{cgaB}$ & 24 & 33.3 & 20.8 & 87.5 & 56.0 & 60.7 & 68.0 & 76.0 & 79.2 & legl_ & 13 & 63.2 & 92.3 & 66.7 & 58.8 & 100 & 53.6 & 90.0 & 69.2 \\
\hline 1 ay7(A:B) & $1 \mathrm{rghB}$ & 15 & 75.0 & 100 & 27.3 & 17.6 & 53.8 & 33.3 & 0 & 0 & $1 \mathrm{a} 19 \mathrm{~B}$ & 15 & 60.0 & 80.0 & 72.7 & 53.3 & 92.9 & 81.2 & 0 & 0 \\
\hline 1cgi(E:I) & $2 \mathrm{cgaB}$ & 33 & 64.3 & 27.3 & 100 & 55.2 & 56.0 & 48.2 & 96.2 & 75.8 & $1 \mathrm{hpt}$ & 19 & 93.3 & 73.7 & 100 & 36.8 & 89.5 & 77.3 & 100 & 63.2 \\
\hline 1d6r(A:I) & $2 \operatorname{tgt}_{-}$ & 27 & 43.8 & 25.9 & 54.5 & 28.6 & 53.6 & 71.4 & 73.9 & 63.0 & $1 \mathrm{~kg} \mathrm{bA}$ & 13 & 66.7 & 92.3 & 44.4 & 53.3 & 35.7 & 15.2 & 22.2 & 15.4 \\
\hline $1 \mathrm{dfj}(\mathrm{E}: \mathrm{I})$ & 9rsaB & 29 & 41.0 & 55.2 & 64.3 & 26.5 & 57.7 & 48.4 & 55.0 & 37.9 & $2 b n h$ & 33 & 43.5 & 60.6 & 81.3 & 31.0 & 32.4 & 91.7 & 21.3 & 30.3 \\
\hline 1 e6e(A:B) & $1 \mathrm{e} 1 \mathrm{nA}$ & 20 & 42.3 & 55.0 & 0 & 0 & 26.9 & 43.8 & 14.9 & 55.0 & $1 c j e D$ & 23 & 65.2 & 65.2 & 93.3 & 50.0 & 79.2 & 73.1 & 15.4 & 17.4 \\
\hline 1 eaw(A:B) & 1eaxA & 22 & 21.1 & 18.2 & 100 & 48.0 & 46.8 & 60.0 & 66.7 & 72.7 & 9pti_ & 14 & 52.6 & 71.4 & 100 & 42.9 & 95.0 & 79.2 & 8.3 & 7.1 \\
\hline 1 ewy(A:C) & 1 gjrA & 19 & 57.1 & 84.2 & 9.1 & 5.3 & 5.6 & 8.3 & 16.7 & 52.6 & 1 czpA & 17 & 51.6 & 94.1 & 57.1 & 42.1 & 63.2 & 63.2 & 50.0 & 41.2 \\
\hline $1 \mathrm{f} 34(\mathrm{~A}: \mathrm{B})$ & 4pep_ & 25 & 44.8 & 52.0 & 30.8 & 12.5 & 30.3 & 52.6 & 47.5 & 76.0 & $1 \mathrm{f} 32 \mathrm{~A}$ & 24 & 57.9 & 45.8 & 72.7 & 24.2 & 55.2 & 69.6 & 70.4 & 79.2 \\
\hline $1 \operatorname{mah}(A: F)$ & 1j06B & 27 & 35.9 & 51.9 & 16.7 & 3.4 & 28.0 & 63.6 & 36.6 & 96.3 & $1 \mathrm{fsc}_{-}$ & 21 & 86.4 & 90.5 & 15.8 & 15.0 & 33.3 & 21.9 & 33.3 & 28.6 \\
\hline $1 \mathrm{ppe}(\mathrm{E}: \mathrm{I})$ & 1 btp_ & 27 & 64.9 & 88.9 & 64.3 & 42.9 & 40.9 & 42.8 & 0 & 0 & $1 l u 0 \mathrm{~A}$ & 14 & 63.2 & 85.7 & 92.3 & 75.0 & 100 & 56.0 & 90.0 & 64.3 \\
\hline $1 \mathrm{tmq}(\mathrm{A}: \mathrm{B})$ & 1jae_ & 28 & 62.2 & 82.1 & 75.0 & 40.0 & 36.0 & 30.0 & 63.4 & 92.9 & 1b1uA & 26 & 57.1 & 76.9 & 93.3 & 56.0 & 70.4 & 76.0 & 0 & 0 \\
\hline 1udi(E:I) & $1 \mathrm{udh}$ & 26 & 52.2 & 46.2 & 63.6 & 25.9 & 48.0 & 66.7 & 72.0 & 69.2 & 2ugiB & 26 & 94.4 & 65.4 & 92.9 & 56.5 & 72.7 & 80.0 & 85.7 & 46.2 \\
\hline $2 p c c(A: B)$ & $1 \mathrm{ccp}$ & 13 & 20.0 & 23.1 & 53.8 & 50.0 & 26.7 & 33.3 & 0 & 0 & $1 y c c_{-}$ & 14 & 26.3 & 35.7 & 42.9 & 35.3 & 37.5 & 33.3 & 13.3 & 14.3 \\
\hline $2 \operatorname{sic}(E: I)$ & 1sup_ & 26 & 50.0 & 46.2 & 72.7 & 38.1 & 81.8 & 60.0 & 62.5 & 76.9 & $3 s s i_{-}$ & 12 & 84.6 & 91.7 & 0 & 0 & 100 & 72.2 & 0 & 0 \\
\hline 2sni(E:I) & 1 ubnA & 27 & 66.7 & 59.3 & 60.0 & 33.3 & 60.0 & 83.0 & 66.7 & 81.5 & $2 \mathrm{ci} 2 \mathrm{l}$ & 15 & 42.9 & 40.0 & 57.1 & 57.1 & 0 & 0 & 76.9 & 66.7 \\
\hline 7cei(A:B) & 1 unkD & 20 & 76.9 & 50.0 & 75.0 & 35.3 & 47.4 & 60.0 & 75.0 & 45.0 & $1 \mathrm{m08B}$ & 16 & 64.3 & 56.3 & 40.0 & 37.5 & 0 & 0 & 13.8 & 25.0 \\
\hline \multicolumn{21}{|l|}{ others } \\
\hline 1ak4(A:D) & $2 \mathrm{cpl} \mathrm{L}_{-}$ & 17 & 42.9 & 35.3 & 50.0 & 31.3 & 33.3 & 18.8 & 59.1 & 76.5 & 1е6jP & 9 & 30.4 & 77.8 & 0 & 0 & 0 & 0 & 0 & 0 \\
\hline 1atn(A:D) & $1 \mathrm{ijj} B$ & 17 & 5.3 & 5.9 & 0 & 0 & 20.7 & 37.5 & 0 & 0 & $3 d n i_{-}$ & 24 & 40.0 & 33.3 & 0 & 0 & 0 & 0 & 66.7 & 66.7 \\
\hline $1 b 6 c(A: B)$ & $1 \mathrm{~d} 6 \mathrm{oA}$ & 20 & 54.3 & 95.0 & 83.3 & 55.6 & 40.0 & 11.1 & 93.3 & 70.0 & 1 iasA & 20 & 44.0 & 55.0 & 54.5 & 25.0 & 31.6 & 25.0 & 0 & 0 \\
\hline 1 buh(A:B) & $1 \mathrm{hcl}-$ & 16 & 68.4 & 81.3 & 0 & 0 & 6.3 & 11.8 & 0 & 0 & $1 \mathrm{dksA}$ & 18 & 75.0 & 83.3 & 58.3 & 38.9 & 36.4 & 22.2 & 100 & 66.7 \\
\hline 1e96(A:B) & $1 \mathrm{mh} 1_{-}$ & 14 & 66.7 & 85.7 & 38.5 & 25.0 & 46.2 & 60.0 & 10.0 & 14.3 & $1 \mathrm{hh} 8 \mathrm{~A}$ & 12 & 73.3 & 91.7 & 41.7 & 35.7 & 45.5 & 35.7 & 0 & 0 \\
\hline $1 \mathrm{fq} 1(\mathrm{~A}: \mathrm{B})$ & $1 \mathrm{fpzF}$ & 16 & 63.2 & 75.0 & 0 & 0 & 0 & 0 & 0 & 0 & $1 \mathrm{~b} 39 \mathrm{~A}$ & 16 & 63.2 & 75.0 & 0 & 0 & 30.0 & 23.1 & 17.1 & 37.5 \\
\hline 1 faj(A:B) & $1 \mathrm{tndC}$ & 21 & 20.7 & 81.0 & 70.6 & 42.9 & 32.3 & 35.7 & 28.6 & 38.1 & IfqiA & 24 & 18.9 & 58.3 & 90.9 & 47.6 & 42.9 & 14.3 & 78.9 & 62.5 \\
\hline $1 \mathrm{gcq}(\mathrm{B}: \mathrm{C})$ & 1 griB & 14 & 35.3 & 42.9 & 70.0 & 63.6 & 38.9 & 63.6 & 22.2 & 14.3 & $1 \mathrm{gcp} B$ & 18 & 78.9 & 83.3 & 60.0 & 40.0 & 100 & 33.3 & 33.3 & 16.7 \\
\hline 1ghq(A:B) & $1 c 3 d_{-}$ & 10 & 41.7 & 100 & 0 & 0 & 42.9 & 37.5 & 0 & 0 & 1 ly $2 \mathrm{~A}$ & 9 & 47.4 & 100 & 0 & 0 & 42.9 & 66.7 & 8.7 & 22.2 \\
\hline $\operatorname{lgrn}(A: B)$ & $1 \mathrm{a} 4 \mathrm{rA}$ & 17 & 54.2 & 76.5 & 33.3 & 15.0 & 40.0 & 40.0 & 50.0 & 58.8 & 1rgp_ & 22 & 50.0 & 54.5 & 16.7 & 4.5 & 100 & 13.6 & 78.9 & 68.2 \\
\hline $1 \mathrm{~h} 1 \mathrm{v}(\mathrm{A}: \mathrm{G})$ & $1 \mathrm{ijj} B$ & 24 & 28.6 & 41.7 & 46.2 & 13.0 & 35.3 & 26.1 & 38.8 & 76.0 & $1 \mathrm{~d} 0 \mathrm{nB}$ & 25 & 43.8 & 56.0 & 0 & 0 & 40.0 & 4.9 & 4.7 & 12.0 \\
\hline
\end{tabular}


Table 5 Detailed Results of DoBi, metaPPI, meta-PPISP and PPI-Pred on 41 complexes (Continued)

\begin{tabular}{|c|c|c|c|c|c|c|c|c|c|c|c|c|c|c|c|c|c|c|c|c|}
\hline 1 he1 (C:A) & $1 \mathrm{mh} 1_{-}$ & 16 & 48.0 & 75.0 & 66.7 & 30.8 & 50.0 & 42.3 & 0 & 0 & 1he9A & 21 & 40.9 & 42.9 & 76.5 & 46.4 & 33.3 & 7.1 & 0 & 0 \\
\hline 1he8(B:A) & $821 P_{-}$ & 13 & 20.6 & 100 & 0 & 0 & 43.8 & 33.3 & 26.7 & 61.5 & $1 \mathrm{e} 8 \mathrm{zA}$ & 15 & 11.1 & 53.3 & 42.9 & 16.7 & 5.9 & 5.6 & 0.6 & 6.7 \\
\hline $1 \mathrm{i} 2 \mathrm{~m}(\mathrm{~A}: \mathrm{B})$ & $19 g 4 A$ & 24 & 14.3 & 33.3 & 42.9 & 21.4 & 43.8 & 50.0 & 15.0 & 12.5 & $1 \mathrm{a} 12 \mathrm{~A}$ & 32 & 15.1 & 43.8 & 0 & 0 & 50.0 & 5.1 & 48.0 & 75.0 \\
\hline 1ibr(A:B) & $19 g 4 A$ & 35 & 43.2 & 45.7 & 73.3 & 22.0 & 55.0 & 22.0 & 14.3 & 8.6 & $1 f 59 A$ & 42 & 38.9 & 33.3 & 7.1 & 1.8 & 0 & 0 & 10.3 & 16.7 \\
\hline $1 \mathrm{kac}(\mathrm{A}: \mathrm{B})$ & 1nobF & 15 & 68.4 & 86.7 & 0 & 0 & 15.4 & 21.1 & 0 & 0 & $1 f 5 w B$ & 21 & 83.3 & 95.2 & 60.0 & 28.6 & 71.4 & 23.8 & 35.3 & 28.6 \\
\hline 1 ktz(A:B) & 1 tgk & 9 & 26.7 & 44.4 & 45.5 & 62.5 & 13.3 & 25.0 & 50.0 & 88.9 & $1 \mathrm{~m} 9 \mathrm{zA}$ & 12 & 57.1 & 100 & 66.7 & 80.0 & 60.0 & 60.0 & 33.3 & 50.0 \\
\hline $1 \mathrm{kxp}(\mathrm{A}: \mathrm{D})$ & $1 \mathrm{ijj} B$ & 34 & 13.6 & 8.8 & 81.3 & 30.2 & 45.5 & 23.3 & 4.3 & 5.9 & $1 \mathrm{kw} 2 \mathrm{~B}$ & 41 & 32.0 & 19.5 & 0 & 0 & 75.0 & 13.0 & 48.9 & 56.1 \\
\hline $1 \mathrm{kxq}(\mathrm{H}: \mathrm{A})$ & $1 \mathrm{kxqH}$ & 25 & 12.1 & 16.0 & 91.7 & 30.6 & 78.6 & 30.6 & 18.2 & 8.0 & $1 \mathrm{ppi}$ & 30 & 22.7 & 16.7 & 41.7 & 17.9 & 20.0 & 3.6 & 47.8 & 73.3 \\
\hline $1 \mathrm{~m} 10(\mathrm{~A}: \mathrm{B})$ & 1auq- & 24 & 57.1 & 50.0 & 58.3 & 24.1 & 65.0 & 44.8 & 50.0 & 45.8 & $1 \mathrm{mozB}$ & 29 & 68.0 & 58.6 & 0 & 0 & 31.6 & 18.2 & 0 & 0 \\
\hline 1qa9(A:B) & $1 \mathrm{hnf} \_$ & 16 & 76.2 & 100 & 0 & 0 & 27.3 & 17.6 & 10.0 & 12.5 & $1 c c z A$ & 16 & 82.4 & 87.5 & 6.7 & 5.3 & 22.2 & 10.5 & 28.6 & 25.0 \\
\hline $1 \mathrm{sbb}(\mathrm{A}: \mathrm{B})$ & $1 \mathrm{bec}_{-}$ & 13 & 54.2 & 100 & 0 & 0 & 17.6 & 17.6 & 0 & 0 & 1 se 4 & 11 & 50.0 & 100 & 0 & 0 & 50.0 & 12.5 & 10.0 & 27.3 \\
\hline $1 w q 1(R: G)$ & $6 q 21 D$ & 26 & 61.5 & 61.5 & 66.7 & 32.3 & 41.7 & 32.2 & 76.2 & 61.5 & 1wer_ & 33 & 62.5 & 45.5 & 100 & 26.5 & 36.4 & 11.8 & 70.0 & 63.6 \\
\hline 2btf(A:P) & $1 \mathrm{ijj} B$ & 26 & 63.3 & 73.1 & 53.3 & 32.0 & 25.0 & 12.0 & 22.0 & 42.3 & 1pne_ & 23 & 56.0 & 60.9 & 0 & 0 & 70.0 & 28.0 & 0 & 0 \\
\hline
\end{tabular}

aPDB is the unbound structure of the two proteins in complex

$\mathrm{b} / n t_{n}$ is the number of residues on actual interface in complex.

${ }^{c} A c c(\%)$ is the accuracy of the corresponding method on the data set.

${ }^{d} \operatorname{Cov}(\%)$ is the coverage of the corresponding method on the data set.

${ }^{e} E-l$ is the type of enzyme-inhibitor.

${ }^{f}$ The values for metaPPI and meta-PPISP are from literatures [14].

9 The results for PPI-Pred are calculated by using the same definition of actual interface with DoBi.

${ }^{h}$ The binding sites between chain $\mathrm{E}$ and chain I of 1acb are predicted by each method; Two unbound structures are chain B of 2cga and the only one chain of 1egl. 
Table 6 Detailed Results of DoBi, metaPPI, meta-PPISP and PPI-Pred on 27 targets

\begin{tabular}{|c|c|c|c|c|c|c|c|c|c|c|c|c|c|c|c|c|c|c|}
\hline \multirow{3}{*}{ Complex } & \multicolumn{9}{|c|}{ Protein 1} & \multicolumn{9}{|c|}{ Protein 2} \\
\hline & \multirow[b]{2}{*}{$I n t_{n}{ }^{a}$} & \multicolumn{2}{|c|}{ DoBi } & \multicolumn{2}{|c|}{ metaPPI $^{d}$} & \multicolumn{2}{|c|}{ meta-PPISP ${ }^{d}$} & \multicolumn{2}{|c|}{ PPI-Pred $^{d}$} & \multirow[b]{2}{*}{$I n t_{\mathrm{n}}$} & \multicolumn{2}{|c|}{ DoBi } & \multicolumn{2}{|c|}{ metaPPI } & \multicolumn{2}{|c|}{ meta-PPISP } & \multicolumn{2}{|c|}{ PPI-Pred } \\
\hline & & $A c c^{\mathrm{b}}$ & $\operatorname{Cov}^{c}$ & Acc & $\operatorname{Cov}$ & $A c c$ & Cov & Acc & $\operatorname{Cov}$ & & Acc & $\operatorname{Cov}$ & $\overline{A c c}$ & $\operatorname{Cov}$ & Acc & $\operatorname{Cov}$ & Acc & Cov \\
\hline T01 & 11 & 46.2 & 54.5 & - & - & 83.3 & 62.5 & - & - & 13 & 38.9 & 53.8 & - & - & 0 & 0 & - & - \\
\hline T02 & 7 & 24.1 & 100 & - & - & 72.2 & 43.3 & - & - & 6 & 21.4 & 100 & - & - & 0 & 0 & - & - \\
\hline T03 & 10 & 12.0 & 30.0 & - & - & 60.0 & 75.0 & - & - & 15 & 32.0 & 53.3 & - & - & 19.6 & 18.0 & - & - \\
\hline T04 & 19 & 50.0 & 89.5 & 0 & 0 & 58.3 & 38.9 & 2.4 & 3.6 & 18 & 37.5 & 100 & 64.3 & 40.9 & 0 & 0 & 71.4 & 68.2 \\
\hline T05 & 20 & 29.2 & 35.0 & 0 & 0 & 52.6 & 33.3 & 4.8 & 9.1 & 17 & 14.3 & 35.3 & 90.0 & 39.1 & 4.5 & 7.7 & 38.9 & 30.4 \\
\hline T06 & 23 & 28.6 & 34.8 & 71.4 & 29.4 & 39.1 & 27.3 & 59.5 & 73.5 & 29 & 38.1 & 27.6 & 28.6 & 15.4 & 25.8 & 66.7 & 4.5 & 3.8 \\
\hline T07 & 15 & 52.9 & 60.0 & 33.3 & 30.8 & 33.3 & 30.8 & 0 & 0 & 11 & 15.4 & 18.2 & 7.7 & 5.6 & 5.6 & 4.3 & 0 & 0 \\
\hline T08 & 25 & 37.9 & 44.0 & 0 & 0 & 9.5 & 8.3 & 0 & 0 & 23 & 64.0 & 69.6 & 30.0 & 11.5 & 0 & 0 & 7.9 & 11.5 \\
\hline T09 & 37 & 90.5 & 51.4 & 80.0 & 20.0 & 0 & 0 & 25.8 & 20.0 & 37 & 76.7 & 62.2 & 45.5 & 12.5 & 0 & 0 & 16.1 & 12.5 \\
\hline T10 & 46 & 40.0 & 47.8 & - & - & 10.0 & 47.4 & - & - & 53 & 50.0 & 49.1 & - & - & 0 & 0 & - & - \\
\hline T11 & 12 & 50.0 & 91.7 & 86.7 & 59.1 & - & - & 45.8 & 50.0 & 28 & 71.9 & 82.1 & 81.8 & 50.0 & - & - & 56.5 & 72.2 \\
\hline T12 & 12 & 16.7 & 25.0 & 93.8 & 62.5 & 61.5 & 30.8 & 45.5 & 41.7 & 28 & 86.4 & 67.9 & 55.6 & 33.3 & 36.0 & 45.0 & 22.2 & 13.3 \\
\hline T13 & 10 & 33.3 & 100 & - & - & 0 & 0 & - & - & 8 & 44.4 & 100 & - & - & 72.0 & 85.7 & - & - \\
\hline T14 & 53 & 52.2 & 22.6 & 10.0 & 2.3 & 6.8 & 33.3 & 8.6 & 7.0 & 63 & 42.3 & 17.5 & 50.0 & 13.2 & 13.5 & 19.2 & 2.0 & 2.6 \\
\hline T15 & 23 & 95.0 & 82.6 & 0 & 0 & 63.2 & 50.0 & 5.0 & 11.1 & 19 & 81.0 & 89.5 & 15.8 & 33.3 & 56.5 & 72.2 & 9.1 & 11.1 \\
\hline T16 & - & - & - & 55.6 & 21.7 & 87.0 & 74.1 & 0 & 0 & - & - & - & 100 & 29.0 & 25.0 & 53.8 & 61.8 & 67.7 \\
\hline T17 & - & - & - & 0 & 0 & 23.1 & 12.5 & 0 & 0 & - & - & - & 92.9 & 65.0 & 0 & 0 & 33.3 & 45.0 \\
\hline T18 & 24 & 53.6 & 62.5 & 85.7 & 50.0 & 42.9 & 36.0 & 46.2 & 50.0 & 31 & 50.0 & 35.5 & 0 & 0 & 52.2 & 36.4 & 2.1 & 3.4 \\
\hline T19 & 12 & 68.8 & 91.7 & - & - & 33.3 & 28.0 & - & - & 12 & 45.0 & 75.0 & - & - & 69.2 & 62.1 & - & - \\
\hline T20 & 47 & 53.6 & 31.9 & 94.4 & 37.8 & 23.8 & 90.9 & 28.6 & 22.2 & 35 & 72.2 & 37.1 & 72.2 & 36.1 & 34.3 & 54.5 & 23.2 & 63.9 \\
\hline T21 & 17 & 73.7 & 82.4 & 0 & 0 & 0 & 0 & 3.0 & 6.7 & 15 & 55.6 & 66.7 & 0 & 0 & 33.3 & 20.8 & 0 & 0 \\
\hline T22 & 17 & 22.7 & 29.4 & 9.1 & 6.7 & 28.6 & 17.4 & 0 & 0 & 12 & 71.4 & 83.3 & 83.3 & 41.7 & 6.2 & 5.9 & 60.0 & 75.0 \\
\hline T23 & 49 & 95.6 & 87.8 & 64.3 & 17.0 & 18.2 & 53.3 & 66.0 & 62.3 & 49 & 95.3 & 83.7 & 64.3 & 17.0 & 0 & 0 & 66.0 & 62.3 \\
\hline T24 & 3 & 13.3 & 66.7 & 66.7 & 66.7 & - & - & 50.0 & 73.3 & 1 & 5.6 & 100 & 0 & 0 & - & - & 50.0 & 61.5 \\
\hline T25 & - & - & - & 100 & 68.2 & 20.0 & 23.5 & 81.8 & 81.8 & - & - & - & 58.3 & 31.8 & 73.9 & 77.3 & 55.6 & 90.9 \\
\hline T26 & 34 & 43.8 & 41.2 & 75.0 & 27.3 & 20.8 & 33.3 & 0 & 0 & 24 & 61.5 & 66.7 & 21.4 & 12.5 & 18.2 & 60.0 & 18.2 & 8.3 \\
\hline T27 & 7 & 43.8 & 87.5 & 0 & 0 & 0 & 0 & 6.7 & 22.2 & 8 & 50.0 & 91.7 & 20.0 & 22.2 & 0 & 0 & 0 & 0 \\
\hline
\end{tabular}

${ }^{\mathrm{b}} \mathrm{Acc}(\%)$ is the accuracy of the corresponding method on the data set.

${ }^{\mathrm{C}} \mathrm{Cov}(\%)$ is the coverage of the corresponding method on the data set.

${ }^{\mathrm{d}}$ The values for these methods are from literatures $[10,14]$. 

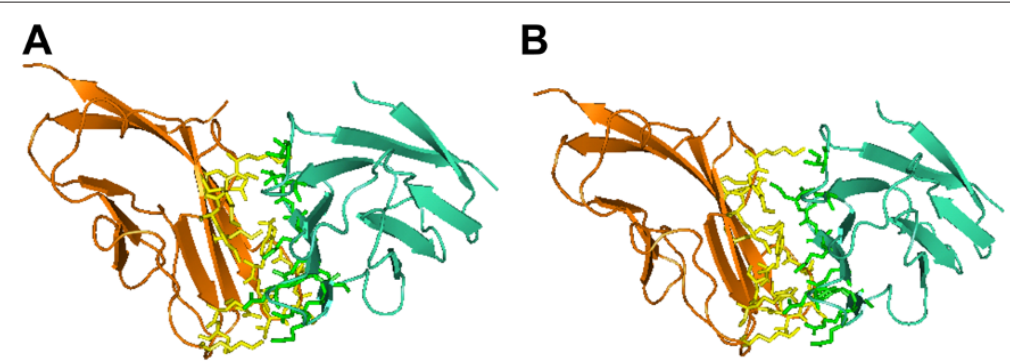

Figure 3 Configuration discovered by DoBi for 1qa9(A:B). (A) is the figuration by DoBi; and (B) is the experimental structure. The $C_{\alpha}$ iRMSD between two complexes is $2.36 \AA$.

have the F-scores $0.35,0.43$ and 0.32 respectively. DoBi has a success rate of $53.7 \%$, as well as overall accuracy and coverage of $50.0 \%$ and $60.0 \%$ respectively.

The detailed results on all the unbound structures of the 41 complexes are displayed in Table 5 . The detailed results on 27 CAPRI targets are displayed in Table 6. Each row displays the results of the methods tested on the two corresponding binding partners.

Besides the identification of binding sites, our program also estimates the orientations and positions of the proteins after binding. Figure 3 displays the orientation and position discovered by our program for $1 \mathrm{qa} 9(\mathrm{~A}: \mathrm{B})$. The $C_{\alpha}$ interface RMSD (root mean squared deviation) (iRMSD) between the experimental structure and the predicted complex is $2.36 \AA$.

\section{Comparison to ProMate and PINUP}

In this experiment, DoBi is compared to ProMate and PINUP. The test data is originally used by ProMate, and consists of 57 non-homologous proteins. The results are reported in Table 7. DoBi has an F-score of 0.56, while PINUP and ProMate have the F-scores 0.43 and 0.21 respectively. The overall accuracy and coverage of DoBi are $54.2 \%$ and $59.1 \%$. The success rate of DoBI is $64.9 \%$. Hence the success rate is improved by at least $1.8 \%$, while the overall accuracy and coverage are improved by at least $1.7 \%$ and $16.6 \%$ respectively.

The average of the sizes predicted by DoBi, PINUP and ProMate are 23.5 residues, 19.0 residues and 5.4 residues respectively, while the actual average size (average size of actual interface residues) is 21.0 residues. The number of residues correctly predicted to be on interface by $\mathrm{DoBi}$, PINUP and ProMate are 12.3 residues, 8.3 residues and 2.7 residues respectively.

Table 8 shows the detailed results of 57 unbound proteins. DoBi performed better for most of the cases. However, for some cases where all three methods do not perform well, DoBi is usually the worst, e.g. 1avu_, 1aye, 1qqrA and 1b1eA.

\section{Comparison to core-SVM}

In this study, we compare DoBi to core-SVM using the same data set of 50 dimers which core-SVM was tested against [12]. The results are shown in Table 9. The overall accuracy and coverage for our method are $59.0 \%$ and $61.1 \%$, while those for core-SVM are $53.4 \%$ and $60.6 \%$. The success rate of $\mathrm{DoBi}$ is $70.0 \%$ on 50 pairs of proteins in those binary complexes. The F-score is 0.60 for $\mathrm{DoBi}$, and 0.56 for core-SVM. The average of the size predicted by $\mathrm{DoBi}$ is 39.0 residues (with standard deviation 19.1), while the average actual size is 40.3 residues. The number of residues correctly predicted by DoBi to be on the interface is 22.5 .

Table 10 shows the details for DoBi on the data set used by core-SVM. The performance of DoBi is particularly good on several proteins such as 1aym2 and 1rzhM.

\section{Evaluation on benchmark v4.0}

To further evaluate our method, we perform tests on the protein-protein docking benchmark v4.0 [32,33]. This

Table 7 Comparison to PINUP and ProMate

\begin{tabular}{|c|c|c|c|c|c|c|c|c|c|c|c|c|c|c|c|c|c|c|}
\hline & \multicolumn{6}{|c|}{ DoBi } & \multicolumn{6}{|c|}{ PINUP } & \multicolumn{6}{|c|}{ ProMate } \\
\hline & Suc $^{a}$ & $\operatorname{Acc}^{b}$ & $\operatorname{Cov}^{c}$ & $F^{f}$ & $M^{\mathrm{d}}$ & $V^{e}$ & Suc & Acc & Cov & $F$ & $M$ & $v$ & Suc & Acc & Cov & $F$ & $M$ & $v$ \\
\hline Overall & 64.9 & 54.2 & 59.1 & 0.56 & 23.5 & 10.5 & 42.1 & 44.9 & 42.5 & 0.43 & 19.0 & 8.7 & 63.1 & 52.5 & 13.2 & 0.21 & 5.4 & 16.8 \\
\hline
\end{tabular}

${ }^{a}$ Suc (\%) is the success rate of the corresponding method on the data set.

${ }^{\mathrm{b}} \mathrm{Acc}(\%)$ is the average accuracy of the corresponding method on the data set.

${ }^{c} \mathrm{Cov}(\%)$ is the average coverage of the corresponding method on the data set.

${ }^{\mathrm{d}} M$ is the average of the sizes predicted by the corresponding method on the data set.

${ }^{\mathrm{e}} V$ is the standard deviation of the sizes predicted by the corresponding method on the data set.

${ }^{f} F$ is the $F$-score of the corresponding method on the data set. 
Table 8 Detailed Comparison to PINUP and ProMate

\begin{tabular}{|c|c|c|c|c|c|c|c|c|}
\hline \multirow[t]{2}{*}{$\mathrm{PDB}^{\mathrm{a}}$} & \multirow[t]{2}{*}{ Complex } & \multirow[t]{2}{*}{$I n t_{n}{ }^{b}$} & \multicolumn{2}{|c|}{ DoBi } & \multicolumn{2}{|c|}{ PINUPg } & \multicolumn{2}{|c|}{ ProMate $^{f}$} \\
\hline & & & $\mathrm{Acc}^{\mathrm{C}}$ & $\operatorname{Cov}^{d}$ & Acc & $\operatorname{Cov}$ & Acc & Cov \\
\hline $1 \mathrm{a} 19 \mathrm{~A}$ & $1 \mathrm{brs}(\mathrm{A}: \mathrm{D})$ & 16 & 86.7 & 81.3 & 72.2 & 81.3 & 100 & 29 \\
\hline $1 \mathrm{a} 2 \mathrm{pA}$ & $1 \mathrm{brs}(\mathrm{D}: \mathrm{A})$ & 19 & 76.2 & 84.2 & 63.6 & 73.7 & 90 & 19 \\
\hline $1 \mathrm{a} 5 \mathrm{e}_{-}$ & $1 \mathrm{bi} 7(\mathrm{~B}: \mathrm{A})$ & 30 & 82.1 & 76.7 & 41.2 & 23.3 & 88 & 10 \\
\hline $1 \mathrm{acl}_{-}$ & $1 \mathrm{fss}(\mathrm{A}: \mathrm{B})$ & 25 & 36.7 & 72.0 & 35.9 & 56.0 & 24 & 14 \\
\hline 1ag6_ & $2 p c f(A: B)$ & 24 & 65.0 & 54.2 & 56.3 & 37.5 & 70 & 16 \\
\hline 1aje_ & 1am4(D:A) & 18 & 57.1 & 22.2 & 60.0 & 33.3 & 72 & 30 \\
\hline 1ajw_ & $1 c c 0(E: A)$ & 9 & 50.0 & 88.9 & 66.7 & 66.7 & 73 & 24 \\
\hline 1aueA & 1 fap (B:A) & 8 & 58.3 & 87.5 & 15.8 & 37.5 & 90 & 35 \\
\hline 1avu_ & $1 \operatorname{avw}(B: A)$ & 15 & 30.0 & 40.0 & 66.7 & 93.3 & 100 & 29 \\
\hline 1aye_ & $1 \mathrm{dtd}(\mathrm{A}: \mathrm{B})$ & 22 & 42.1 & 36.4 & 44.4 & 54.5 & 54 & 24 \\
\hline 1b1eA & 1a4y(B:A) & 32 & 38.7 & 37.5 & 88.2 & 46.9 & 69 & 24 \\
\hline 1 bip_ & $1 \mathrm{tmq}(\mathrm{B}: \mathrm{A})$ & 29 & 66.7 & 55.2 & 61.1 & 37.9 & 100 & 27 \\
\hline $1 \mathrm{ctm}-$ & $2 p c f(B: A)$ & 21 & 62.1 & 85.7 & 38.1 & 38.1 & 100 & 12 \\
\hline 1cto_ & $1 \mathrm{~cd} 9(\mathrm{~B}: \mathrm{A})$ & 6 & 40.0 & 33.3 & 35.3 & 100 & 36 & 29 \\
\hline 1cye_ & 1eay(A:B) & 16 & 55.6 & 62.5 & 5.6 & 6.3 & 0 & 0 \\
\hline $1 \mathrm{~d} 0 \mathrm{nA}$ & $1 \mathrm{cof}(\mathrm{S}: \mathrm{A})$ & 27 & 46.2 & 44.4 & 0 & 0 & 67 & 3 \\
\hline $1 \mathrm{~d} 2 \mathrm{bA}$ & 1 uea(B:A) & 19 & 66.7 & 52.6 & 78.6 & 57.9 & 92 & 31 \\
\hline 1ekxA & $1 \mathrm{~d} 09(\mathrm{~A}: \mathrm{B})$ & 21 & 64.5 & 95.2 & 0 & 0 & 0 & 0 \\
\hline $1 \mathrm{ex} 3 \mathrm{~A}$ & $1 \mathrm{cgi}(\mathrm{E}: \mathrm{I})$ & 33 & 61.1 & 33.3 & 68.2 & 45.5 & 100 & 29 \\
\hline $1 \mathrm{ez} 3 \mathrm{~A}$ & $1 \mathrm{dn} 1(\mathrm{~B}: \mathrm{A})$ & 18 & 88.9 & 44.4 & 47.1 & 44.4 & 100 & 6 \\
\hline 1eza. & $3 e z a(A: B)$ & 21 & 64.0 & 76.2 & 0 & 0 & 0 & 0 \\
\hline 1eztA & 1agr(E:A) & 22 & 57.1 & 54.5 & 22.2 & 18.2 & 54 & 13 \\
\hline 1fool & 1f02(l:T) & 17 & 31.6 & 35.3 & 0 & 0 & 0 & 0 \\
\hline $1 f 5 w A$ & $1 \mathrm{kac}(\mathrm{B}: \mathrm{A})$ & 21 & 71.4 & 71.4 & 25.0 & 23.8 & 100 & 6 \\
\hline $1 \mathrm{fkl}$ & $1 \mathrm{~b} 6 \mathrm{c}(\mathrm{A}: \mathrm{B})$ & 19 & 54.5 & 63.2 & 75.0 & 47.4 & 100 & 20 \\
\hline $1 \mathrm{flzA}$ & 1eui(A:C) & 25 & 42.9 & 96.0 & 77.3 & 68.0 & 52 & 19 \\
\hline $1 \mathrm{fvhA}$ & $1 \mathrm{dn} 1(\mathrm{~A}: \mathrm{B})$ & 42 & 51.4 & 45.2 & 53.3 & 38.1 & 0 & 0 \\
\hline $1 \mathrm{~g} 4 \mathrm{kA}$ & 1 uea(A:B) & 30 & 46.2 & 40.0 & 43.8 & 23.3 & 78 & 21 \\
\hline $1 \mathrm{gc} 7 \mathrm{~A}$ & 1 ef1 $(A: C)$ & 18 & 71.4 & 55.6 & 28.6 & 11.1 & 78 & 6 \\
\hline 1gnc. & $1 \mathrm{~cd} 9(\mathrm{~A}: \mathrm{B})$ & 15 & 43.7 & 46.7 & 21.4 & 20.0 & 6 & 2 \\
\hline $1 \mathrm{hh} 8 \mathrm{~A}$ & 1e96(B:A) & 14 & 50.0 & 35.7 & 44.0 & 78.6 & 50 & 2 \\
\hline $1 \mathrm{hplA}$ & 1eth(A:B) & 19 & 20.0 & 36.8 & 8.7 & 10.5 & 7 & 3 \\
\hline 1hu8A & $1 y \operatorname{cs}(A: B)$ & 8 & 37.5 & 75.0 & 31.6 & 75.0 & 5 & 2 \\
\hline 1iob_ & $1 \mathrm{itb}(\mathrm{A}: \mathrm{B})$ & 38 & 38.1 & 21.1 & 46.7 & 18.4 & 31 & 6 \\
\hline 1j6zA & $1 \mathrm{cof}(\mathrm{A}: \mathrm{S})$ & 29 & 28.2 & 75.9 & 34.6 & 31.0 & 0 & 0 \\
\hline 1jae_ & $1 \mathrm{tmq}(\mathrm{A}: \mathrm{B})$ & 32 & 60.0 & 65.6 & 83.3 & 46.9 & 50 & 13 \\
\hline $1 \mathrm{lba}$ & $1 \operatorname{aro}(L: P)$ & 16 & 8.6 & 18.8 & 40.0 & 37.5 & 60 & 24 \\
\hline 1nobA & $1 \mathrm{kac}(\mathrm{A}: \mathrm{B})$ & 15 & 50.0 & 73.3 & 0 & 0 & 7 & 3 \\
\hline 1 nos & 1 noc(A:B) & 9 & 33.3 & 44.4 & 0 & 0 & 0 & 0 \\
\hline $1 \mathrm{pco}$ & 1eth(B:A) & 15 & 77.8 & 46.7 & 16.7 & 20.0 & 60 & 12 \\
\hline 1pne_ & 1hlu(P:A) & 25 & 65.7 & 92.0 & 93.8 & 60.0 & 0 & 0 \\
\hline 1poh_ & $\operatorname{lggr}(B: A)$ & 10 & 57.1 & 40.0 & 72.7 & 80.0 & 0 & 0 \\
\hline $1 \mathrm{ppp}$ & 1stf(E:I) & 29 & 79.3 & 79.3 & 47.4 & 31.0 & 91 & 30 \\
\hline
\end{tabular}


Table 8 Detailed Comparison to PINUP and ProMate (Continued)

\begin{tabular}{|c|c|c|c|c|c|c|c|}
\hline 1qqrA & $1 \mathrm{bml}(\mathrm{C}: \mathrm{A})$ & 7 & 33.3 & 28.6 & 38.5 & 71.4 & 85 \\
\hline 1rgp_ & 1am4(A:D) & 16 & 55.0 & 68.8 & 36.8 & 43.8 & 50 \\
\hline 1 selA & 1cse(E:I) & 29 & 75.0 & 93.1 & 60.9 & 48.3 & 61 \\
\hline 1 vin_ & 1 fin(B:A) & 29 & 40.0 & 34.5 & 50.0 & 51.7 & 0 \\
\hline 1 wer & 1 wq1 (G:R) & 33 & 67.7 & 63.6 & 70.6 & 36.4 & 0 \\
\hline $1 \times p b_{-}$ & $1 \mathrm{jtg}(\mathrm{A}: \mathrm{B})$ & 32 & 69.2 & 56.3 & 89.5 & 53.1 & 0 \\
\hline $2 \mathrm{bnh}$ & $1 \mathrm{a} 4 \mathrm{y}(\mathrm{A}: \mathrm{B})$ & 38 & 38.5 & 39.5 & 37.8 & 36.8 & 100 \\
\hline $2 \mathrm{cpl} \mathrm{-}_{-}$ & 1ak4(A:D) & 17 & 61.9 & 76.5 & 78.6 & 64.7 & 76 \\
\hline $2 f 3 g A$ & $1 \operatorname{ggr}(A: B)$ & 18 & 50.0 & 50.0 & 64.7 & 61.1 & 100 \\
\hline 2nef. & $1 \operatorname{avz}(B: A)$ & 10 & 56.3 & 90.0 & 30.8 & 40.0 & 57 \\
\hline $2 \mathrm{rgf}_{-}$ & $1 \operatorname{lfd}(A: B)$ & 14 & 52.4 & 78.6 & 27.8 & 35.7 & 20 \\
\hline $3 \mathrm{ssi}_{-}$ & $2 \operatorname{sic}(\mid: E)$ & 15 & 80.0 & 80.0 & 68.2 & 100 & 100 \\
\hline $6 \mathrm{ccp}$ & $2 p c b(A: B)$ & 9 & 23.5 & 44.4 & 28.6 & 66.7 & 0 \\
\hline Bound $^{e}$ & 1jtg(B:A) & 32 & 81.1 & 93.8 & 65.0 & 40.6 & 94 \\
\hline
\end{tabular}

aPDB is the unbound structure of the predicted protein.

${ }^{\mathrm{b}} / n t_{n}$ is the number of residues on actual interface in complex.

${ }^{C} A c c(\%)$ is the accuracy of the corresponding method on the data set.

${ }^{\mathrm{d}} \mathrm{Cov}(\%)$ is the coverage of the corresponding method on the data set.

e The unbound structure of $1 \mathrm{jtgB}$ was not available in PDB, and we used the bound structure instead.

f The values for ProMate are from literature [9].

${ }^{9}$ The results for PINUP are calculated by using the same definition of actual interface with DoBi.

benchmark consists of 176 complexes. Proteins dynamically change their conformations upon binding with other proteins [34]. A single protein without binding with any other structure is referred to as unbound, whereas a protein with a binding partner in a complex is referred to as bound. We test our method in both the bound and the unbound cases.

\section{Running time}

We used a Pentium(R) 4 (CPU of $3.40 \mathrm{GHz}$ ) to run DoBi. The computation for each of the 176 complexes took 100 seconds on average.

\section{Results on bound states}

The complexes are classified into broad biochemical categories: Enzyme-Inhibitor (52), Antibody-Antigen (25) and
Others (99). The average accuracy and coverage of DoBi are $61.8 \%$ and $67.9 \%$ respectively on the 52 complexes in Enzyme-Inhibitor, $51.6 \%$ and $70.1 \%$ on the 25 complexes in Antibody-Antigen, and $58.2 \%$ and $69.1 \%$ on the 99 complexes in Others. A success rate of $77.6 \%$ is achieved for the Enzyme-Inhibitor complexes. The details are shown in Table 11.

\section{Results on unbound states}

The pairs of unbound proteins are classified into three categories: 121 rigid-body (easy) cases, 30 medium difficult cases, and 25 difficult cases, according to the magnitude of conformational change after binding [30]. The average accuracy and coverage of DoBi are $43.6 \%$ and $65.4 \%$ on the 121 rigid-body cases, $34.1 \%$ and $56.7 \%$ on the 30 medium difficult cases, and $32.4 \%$ and $53.4 \%$ on the 25 difficult

Table 9 Comparison to core-SVM

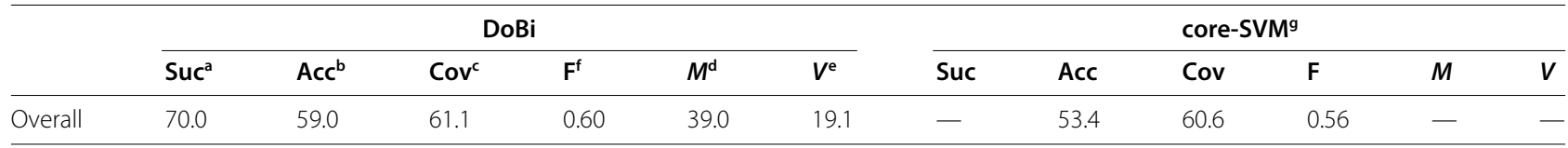

a Suc (\%) is the success rate of the corresponding method on the data set.

${ }^{b} \mathrm{Acc}(\%)$ is the average accuracy of the corresponding method on the data set.

${ }^{\mathrm{C}} \mathrm{Cov}(\%)$ is the average coverage of the corresponding method on the data set

${ }^{\mathrm{d}} M$ is the average predicted size for DoBi on the data set.

${ }^{e} V$ is the standard deviation of predicted size for DoBi on the data set.

${ }^{f} F$ is the F-score of the corresponding method on the data set.

9The values for core-SVM are from literature [12] 
Table 10 Detailed Results for DoBi on the data set used by core-SVM

\begin{tabular}{|c|c|c|c|c|c|c|}
\hline Protein ID & Partner ID & $I n t_{n}{ }^{a}$ & $C_{n}{ }^{b}$ & $P_{n}^{c}$ & $A c c^{d}$ & $\operatorname{Cov}^{\mathrm{e}}$ \\
\hline 1a9xA & $1 \mathrm{a} 9 \mathrm{xB}$ & 59 & 52 & 95 & 54.7 & 88.1 \\
\hline $1 \mathrm{a} 9 \mathrm{xB}$ & 1a9xA & 52 & 47 & 88 & 53.4 & 90.4 \\
\hline 1aym1 & 1aym3 & 46 & 38 & 41 & 92.7 & 82.6 \\
\hline 1aym2 & 1aym1 & 57 & 54 & 70 & 77.1 & 94.7 \\
\hline 1aym3 & 1aym1 & 43 & 33 & 36 & 91.7 & 76.7 \\
\hline $1 \mathrm{blxA}$ & $1 \mathrm{blxB}$ & 21 & 15 & 33 & 45.5 & 71.4 \\
\hline $1 \mathrm{fzcB}$ & $1 \mathrm{fzcC}$ & 45 & 38 & 58 & 65.5 & 84.4 \\
\hline $1 \mathrm{~g} 4 \mathrm{yR}$ & 1g4yB & 29 & 5 & 18 & 27.8 & 17.2 \\
\hline $1 \mathrm{gk} 8 \mathrm{~A}$ & 1gk8l & 49 & 28 & 55 & 50.9 & 57.1 \\
\hline $1 \mathrm{~h} 1 \mathrm{rB}$ & $1 \mathrm{~h} 1 \mathrm{rA}$ & 33 & 9 & 14 & 64.3 & 27.2 \\
\hline $1 \mathrm{~h} 8 \mathrm{ec}$ & $1 \mathrm{~h} 8 \mathrm{eD}$ & 69 & 37 & 67 & 55.2 & 53.6 \\
\hline $1 \mathrm{~h} 8 \mathrm{eD}$ & $1 \mathrm{~h} 8 \mathrm{ec}$ & 35 & 19 & 39 & 48.7 & 54.3 \\
\hline $1 \mathrm{~h} \times s 4$ & 1gxs1 & 31 & 21 & 35 & 60.0 & 67.7 \\
\hline $1 \mathrm{irdB}$ & $1 \operatorname{ird} A$ & 23 & 20 & 32 & 62.5 & 86.9 \\
\hline $1 \mathrm{j} 34 \mathrm{~A}$ & 1j34B & 43 & 19 & 22 & 86.4 & 44.1 \\
\hline 1jboB & 1jboA & 36 & 16 & 29 & 55.2 & 44.4 \\
\hline $1 \mathrm{jsd} A$ & $1 \mathrm{jsdB}$ & 51 & 18 & 20 & 90.0 & 35.3 \\
\hline $1 \mathrm{jsdB}$ & $1 \mathrm{jsdA}$ & 67 & 26 & 42 & 61.9 & 38.8 \\
\hline $1 \mathrm{k} 5 \mathrm{nA}$ & $1 \mathrm{k} 5 \mathrm{nB}$ & 35 & 24 & 56 & 42.9 & 68.6 \\
\hline $1 \mathrm{k} 5 \mathrm{nB}$ & $1 \mathrm{k} 5 \mathrm{nA}$ & 25 & 16 & 39 & 41.0 & 64.0 \\
\hline $1 \mathrm{ld} 8 \mathrm{~A}$ & $11 \mathrm{~d} 8 \mathrm{~B}$ & 35 & 23 & 28 & 82.1 & 65.7 \\
\hline $1 \mathrm{mty} B$ & $1 \mathrm{mty} D$ & 58 & 22 & 34 & 64.7 & 38.1 \\
\hline $1 \mathrm{mty} D$ & $1 \mathrm{mty} B$ & 31 & 10 & 15 & 66.7 & 32.2 \\
\hline $1 \mathrm{mtyG}$ & $1 \mathrm{mty} D$ & 41 & 18 & 42 & 42.9 & 43.9 \\
\hline $1 \mathrm{n} 4 \mathrm{qB}$ & $1 \mathrm{n} 4 \mathrm{qA}$ & 25 & 5 & 15 & 33.3 & 20.0 \\
\hline 1p2jA & 1p2jl & 23 & 18 & 36 & 50.0 & 78.2 \\
\hline 1p2jl & $1 \mathrm{p} 2 \mathrm{jA}$ & 14 & 13 & 21 & 61.9 & 92.9 \\
\hline 1qopA & 1qopB & 35 & 32 & 52 & 61.5 & 91.4 \\
\hline 1qopB & 1qopA & 34 & 31 & 51 & 60.8 & 91.2 \\
\hline 1rthA & 1 rthB & 57 & 32 & 68 & 47.0 & 56.1 \\
\hline 1rthB & 1rthA & 58 & 33 & 69 & 47.8 & 56.9 \\
\hline 1rypB & 1 rypA & 31 & 13 & 24 & 54.1 & 41.9 \\
\hline 1rzhH & $1 \mathrm{rzhM}$ & 37 & 8 & 16 & 50.0 & 21.6 \\
\hline 1rzhL & 1rzhM & 48 & 42 & 45 & 93.3 & 87.5 \\
\hline 1rzhM & $1 \mathrm{rzhL}$ & 51 & 45 & 48 & 93.8 & 88.2 \\
\hline $1 \mathrm{~s} 5 \mathrm{dD}$ & $1 \mathrm{~s} 5 \mathrm{dA}$ & 4 & 4 & 29 & 13.7 & 100 \\
\hline 1 tugA & 1 tugB & 17 & 14 & 39 & 35.9 & 82.4 \\
\hline 1tugB & 1tugA & 12 & 9 & 24 & 37.5 & 75.0 \\
\hline $1 \mathrm{t} \times 4 \mathrm{~B}$ & $1 \mathrm{t} \times 4 \mathrm{~A}$ & 25 & 18 & 34 & 52.9 & 72.0 \\
\hline $1 \mathrm{uvqA}$ & $1 \mathrm{uvqB}$ & 61 & 35 & 39 & 89.7 & 57.4 \\
\hline $1 \mathrm{uvqB}$ & $1 \mathrm{uvqA}$ & 55 & 26 & 31 & 83.9 & 47.2 \\
\hline 1 we $3 F$ & 1we3T & 12 & 10 & 48 & 20.8 & 83.3 \\
\hline $1 \mathrm{wf40}$ & 1 wf4a & 10 & 10 & 19 & 52.6 & 100 \\
\hline
\end{tabular}


Table 10 Detailed Results for DoBi on the data set used by core-SVM (Continued)

\begin{tabular}{|c|c|c|c|c|c|c|}
\hline $2 \operatorname{tnn} A$ & $2 \operatorname{lnn} B$ & 55 & 12 & 16 & 75.0 & 21.8 \\
\hline $2 \mid \operatorname{tn} B$ & $21 \operatorname{tn} A$ & 47 & 17 & 17 & 100 & 36.2 \\
\hline $3 p c g A$ & $3 p c g M$ & 41 & 12 & 15 & 80.0 & 29.3 \\
\hline $3 p c g M$ & $3 p c g A$ & 40 & 11 & 21 & 52.4 & 27.5 \\
\hline 4ubpA & $4 \mathrm{ubpc}$ & 24 & 8 & 43 & 18.6 & 33.3 \\
\hline $4 u b p c$ & $4 \mathrm{ubpB}$ & 46 & 26 & 86 & 30.2 & 56.5 \\
\hline 8rucl & 8rucA & 38 & 29 & 38 & 76.3 & 76.3 \\
\hline
\end{tabular}

${ }^{\mathrm{a}} / n t_{n}$ is the number of residues on actual interface in complex.

${ }^{\mathrm{b}} C_{n}$ is the number of residues correctly predicted to be on interface by our method.

${ }^{c} P_{n}$ is the number of total residues predicted to be on interface by our method.

${ }^{d}$ Acc (\%) is the accuracy of our method on the data set.

${ }^{e} \mathrm{Cov}(\%)$ is the coverage of our method on the data set.

Table 11 DoBi's performance for proteins of benchmark v4.0 in bound states

\begin{tabular}{|c|c|c|c|c|c|c|}
\hline Type $^{a}$ & No. of complexes & Suc $^{\mathrm{b}}$ & $\mathrm{Acc}^{\mathrm{c}}$ & $\operatorname{Cov}^{d}$ & $M^{\mathrm{e}}$ & $v^{f}$ \\
\hline Enzyme-Inhibitor & 52 & 77.6 & 61.8 & 67.9 & 22.6 & 6.3 \\
\hline Antibody-Antigen & 25 & 56.0 & 51.6 & 70.1 & 19.3 & 6.5 \\
\hline Others & 99 & 66.7 & 58.2 & 69.1 & 24.0 & 10.8 \\
\hline Overall & 176 & 68.2 & 57.5 & 68.9 & 22.9 & 9.3 \\
\hline
\end{tabular}

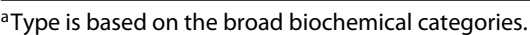

${ }^{b} \mathrm{Suc}(\%)$ is the success rate of $\mathrm{DoBi}$ on the data set.

${ }^{\mathrm{C}} \mathrm{Acc}(\%)$ is the average accuracy of DoBi on the data set.

${ }^{\mathrm{d}} \mathrm{Cov}(\%)$ is the average coverage of DoBi on the data set.

${ }^{\mathrm{e}} M$ is the average of the sizes predicted by DoBi on the data set.

${ }^{f} V$ is the standard deviation of the sizes predicted by DoBi on the data set.

Table 12 DoBi's performance for proteins of benchmark v4.0 in unbound states

\begin{tabular}{|c|c|c|c|c|c|c|c|}
\hline Subset $^{a}$ & Type $^{\text {b }}$ & No. of cases & Suc & $A c c^{d}$ & $\operatorname{Cov}^{\mathrm{e}}$ & $M^{f}$ & $V^{g}$ \\
\hline \multirow[t]{4}{*}{ Rigid body } & Enzyme-Inhibitor & 40 & 51.2 & 48.9 & 66.9 & 37.1 & 34.1 \\
\hline & Antibody-Antigen & 22 & 50.0 & 51.0 & 67.8 & 24.0 & 14.6 \\
\hline & Others & 59 & 32.2 & 37.3 & 63.5 & 39.9 & 36.9 \\
\hline & Subtotal & 121 & 41.7 & 43.6 & 65.4 & 36.1 & 31.9 \\
\hline \multirow[t]{4}{*}{ Medium difficult } & Enzyme-Inhibitor & 7 & 39.9 & 36.7 & 56.2 & 25.9 & 17.4 \\
\hline & Antibody-Antigen & 1 & 0 & 31.9 & 41.4 & 38.0 & 9.2 \\
\hline & Others & 22 & 31.2 & 33.4 & 56.7 & 52.9 & 56.7 \\
\hline & Subtotal & 30 & 31.6 & 34.1 & 56.7 & 46.1 & 45.9 \\
\hline \multirow[t]{4}{*}{ Difficult } & Enzyme-Inhibitor & 5 & 37.5 & 43.1 & 46.5 & 26.1 & 7.0 \\
\hline & Antibody-Antigen & 2 & 0 & 29.5 & 54.6 & 27.3 & 17.5 \\
\hline & Others & 18 & 10.5 & 30.5 & 54.8 & 54.9 & 44.8 \\
\hline & Subtotal & 25 & 13.9 & 32.4 & 53.4 & 46.9 & 35.1 \\
\hline Overall & & 176 & 36.0 & 40.4 & 62.2 & 39.3 & 36.9 \\
\hline
\end{tabular}

a Subset is based on the magnitude of conformational change after binding.

${ }^{\mathrm{b}}$ Type is based on the broad biochemical categories.

'Suc (\%) is the success rate of DoBi on the data set.

${ }^{\mathrm{d}} \mathrm{Acc}(\%)$ is the average accuracy of DoBi on the data set.

${ }^{e} \mathrm{Cov}(\%)$ is the average coverage of $\mathrm{DoBi}$ on the data set.

${ }^{f} M$ is the average predicted size for DoBi on the data set.

$9 V$ is the standard deviation of predicted size for DoBi on the data set. 

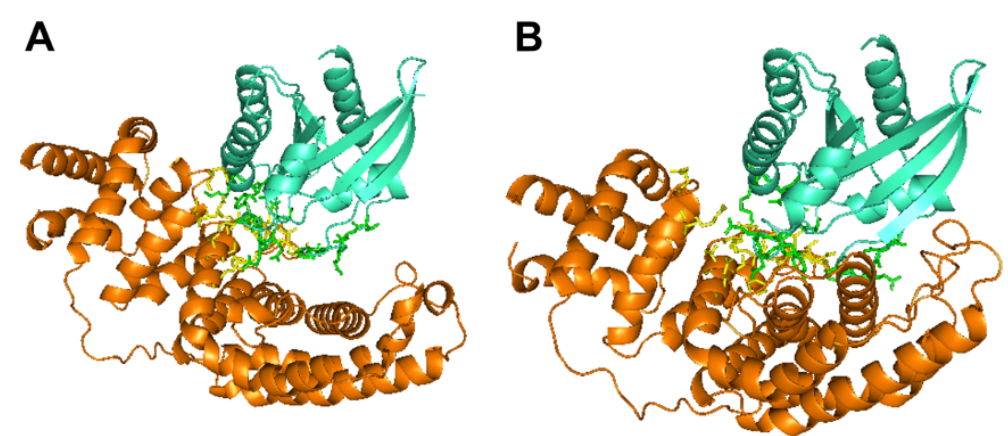

Figure 4 Configuration discovered by DoBi for $\mathbf{1}$ wq1(R:G). (A) is the configuration by DoBi; and $(\mathbf{B})$ is the experimental structure. The $C_{\alpha}$ iRMSD between two complexes is $4.12 \AA$.

Table 13 The Docking Results of DoBi, ZDOCK and 3D-Dock on CAPRI

\begin{tabular}{|c|c|c|c|c|c|c|c|c|c|c|c|c|c|c|}
\hline \multirow[t]{2}{*}{ Target } & \multicolumn{4}{|c|}{$\mathrm{DoBi}_{1000}$} & \multicolumn{4}{|c|}{ ZDOCK } & \multicolumn{4}{|c|}{$\mathrm{DoBi}_{10}$} & \multicolumn{2}{|c|}{ 3D-Dock ${ }^{\mathrm{e}}$} \\
\hline & iRMSD $^{a}$ & $N C^{b}$ & $\mathrm{~F}_{l}^{\mathrm{c}}$ & $F_{r}^{d}$ & iRMSD & $\mathrm{NC}$ & $F_{l}$ & $\mathrm{~F}_{r}$ & iRMSD & $\mathrm{NC}$ & $F_{I}$ & $\mathrm{~F}_{r}$ & iRMSD & NC \\
\hline $\mathrm{T} 1$ & 4.28 & 27.6 & 56.0 & 45.2 & 8.10 & 17.2 & 50.0 & 32.0 & 5.45 & 44.0 & 74.1 & 64.3 & 3.0 & 46 \\
\hline $\mathrm{T} 2$ & 6.23 & 76.9 & 38.8 & 35.3 & 4.15 & 46.2 & 51.9 & 35.7 & 8.27 & 53.8 & 48.0 & 36.4 & - & - \\
\hline T3 & 18.48 & 9.4 & 17.1 & 43.9 & 3.89 & 62.5 & 64.0 & 60.6 & 18.51 & 12.0 & 22.9 & 51.4 & - & - \\
\hline $\mathrm{T} 4$ & 3.98 & 63.5 & 66.6 & 57.1 & 4.50 & 23.1 & 78.2 & 58.3 & 6.24 & 35.9 & 38.3 & 51.6 & 15.1 & 21 \\
\hline T5 & 11.06 & 7.7 & 46.8 & 31.6 & 10.08 & 5.4 & 76.6 & 18.9 & 11.06 & 7.7 & 46.8 & 31.6 & - & - \\
\hline T6 & 16.49 & 15.4 & 36.4 & 33.4 & 8.72 & 29.2 & 54.2 & 71.6 & 19.21 & 9.6 & 18.2 & 28.1 & 0.8 & 86 \\
\hline $\mathrm{T7}$ & 11.10 & 13.5 & 62.8 & 24.0 & 6.43 & 2.7 & 44.4 & 4.8 & 11.10 & 13.5 & 62.8 & 24.0 & 28.6 & 14 \\
\hline T8 & 6.69 & 37.9 & 42.7 & 60.9 & 2.73 & 63.6 & 82.8 & 60.0 & 6.69 & 37.9 & 42.7 & 60.9 & 1.7 & 33 \\
\hline T9 & 2.85 & 33.3 & 61.3 & 67.6 & 8.46 & 28.9 & 54.1 & 58.7 & 10.54 & 1.4 & 36.7 & 37.7 & 9.7 & 23 \\
\hline T10 & 4.52 & 28.9 & 50.4 & 51.8 & 14.75 & 5.9 & 15.4 & 17.3 & 7.69 & 13.0 & 58.1 & 59.3 & 34.8 & 0 \\
\hline T11 & 2.55 & 66.7 & 68.5 & 75.0 & 2.63 & 61.1 & 96.0 & 82.1 & 12.17 & 0 & 0 & 45.0 & 1.9 & 20 \\
\hline T12 & 2.55 & 66.7 & 68.5 & 75.0 & 2.31 & 81.5 & 75.9 & 88.9 & 12.17 & 0 & 0 & 45.0 & 3.2 & 22 \\
\hline T13 & 3.33 & 94.1 & 74.1 & 69.6 & 2.49 & 57.1 & 52.9 & 59.3 & 3.33 & 94.1 & 74.1 & 69.6 & 6.4 & 6 \\
\hline T14 & 19.98 & 9.6 & 34.5 & 28.0 & 5.22 & 42.0 & 72.7 & 68.9 & 20.97 & 10.3 & 36.1 & 28.3 & 0.9 & 47 \\
\hline T15 & 2.40 & 53.6 & 86.9 & 83.0 & 0.86 & 91.1 & 90.6 & 81.8 & 4.00 & 42.0 & 64.2 & 63.6 & - & - \\
\hline T18 & 8.08 & 25.0 & 57.7 & 44.4 & 1.88 & 66.2 & 80.0 & 80.0 & 11.38 & 8.2 & 10.3 & 19.7 & 9.4 & 14 \\
\hline T19 & 2.74 & 58.8 & 60.0 & 69.0 & 9.81 & 4.8 & 40.0 & 14.6 & 2.74 & 58.8 & 60.0 & 69.0 & 3.9 & 31 \\
\hline T20 & 15.13 & 1.1 & 14.7 & 28.6 & 13.62 & 7.2 & 35.0 & 37.1 & 15.13 & 1.1 & 14.7 & 28.6 & - & - \\
\hline T21 & 2.02 & 50.0 & 77.8 & 68.8 & 2.43 & 70.7 & 83.3 & 70.6 & 2.02 & 50.0 & 77.8 & 68.8 & - & - \\
\hline T22 & 16.08 & 7.5 & 20.0 & 71.4 & 9.28 & 12.6 & 66.7 & 0 & 16.08 & 7.5 & 20.0 & 71.4 & - & - \\
\hline T23 & 1.90 & 61.2 & 86.9 & 88.4 & 2.14 & 72.1 & 87.3 & 87.9 & 3.14 & 46.0 & 83.1 & 83.1 & - & - \\
\hline T24 & 5.01 & 50.0 & 31.6 & 20.0 & 28.15 & 0 & 0 & 0 & 5.01 & 50.0 & 31.6 & 20.0 & - & - \\
\hline T26 & 7.11 & 29.6 & 26.1 & 45.2 & 30.07 & 0 & 0 & 0 & 7.11 & 29.6 & 26.1 & 45.2 & - & - \\
\hline $\mathrm{T} 27$ & 6.95 & 60.0 & 42.4 & 51.9 & 15.89 & 3.5 & 24.4 & 0 & 7.38 & 66.7 & 38.5 & 50.0 & - & - \\
\hline T29 & 2.46 & 68.6 & 83.3 & 79.3 & 3.90 & 58.6 & 77.4 & 72.1 & 3.80 & 32.7 & 69.4 & 77.8 & - & - \\
\hline
\end{tabular}

${ }^{\mathrm{a}} C_{\alpha}$ iRMSD between the configuration by the respective method and the experimental structure.

${ }^{\mathrm{b}} N C(\%)$ is fraction of native contacts for each method.

${ }^{{ }^{C}} F_{/}(\%)$ is the $\mathrm{F}$-score of each method for the ligand protein on the data set.

${ }^{d} F_{r}(\%)$ is the $F$-score of each method for the receptor protein on the data set.

eThe values for 3D-Dock are from literatures [36,37]; The blank results mean that 3D-Dock never produced on these targets. 
Table 14 The Docking Results of DoBi and ZDOCK on Benchmark v4.0

\begin{tabular}{|c|c|c|c|c|c|c|c|c|c|c|c|c|c|c|c|c|c|c|c|c|}
\hline \multirow[b]{2}{*}{ PDB } & \multicolumn{3}{|c|}{ DoBi $_{1000}$} & \multicolumn{3}{|c|}{ ZDO } & \multirow[b]{2}{*}{ PDB } & \multicolumn{3}{|c|}{$\operatorname{DoBi}_{1000}$} & \multicolumn{3}{|c|}{ ZDOCK } & \multirow[b]{2}{*}{ DB } & \multicolumn{3}{|c|}{ DoBi $_{1000}$} & \multicolumn{3}{|c|}{ ZDOCK } \\
\hline & mola & $\mathrm{F}_{l^{\mathrm{b}}}^{\mathrm{b}}$ & c & Rmsd & $\mathrm{F}_{I}$ & $\mathrm{~F}$ & & nsd & $F_{I}$ & $F_{r}$ & iRmsd & $F_{l}$ & $\mathrm{~F}_{r}$ & & isd & $F_{l}$ & $\mathrm{~F}_{r}$ & iRmsd & $\mathrm{F}_{I}$ & $\mathrm{~F}_{r}$ \\
\hline vk & & & & & & 0 & 1. & 4 & 7 & 8 & & 3 & 6 & $1 \mathrm{gla}$ & & 7.3 & 72.4 & & .3 & 72. \\
\hline sni & & & 82.8 & & & & & & & & & & & & & 8 & & & 93.8 & \\
\hline $2 j$ & 1.52 & 0 & .9 & & 7 & 5.4 & & 4 & 7 & 1.2 & & & 3.5 & 25 & & .2 & 68.6 & & 0.0 & 72 \\
\hline 1 & 1. & 88.5 & 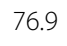 & 182 & 77.6 & 2 & $1 \mathrm{fq} 1$ & 4 & 62.9 & 76.5 & 8.05 & 42.4 & 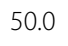 & k & 6.60 & 7 & 2 & & 3 & 75 \\
\hline 10 & 1.68 & 80.0 & 2 & & 0 & & & & & 60.9 & & 0 & & 2 & & 5 & 7 & & .0 & \\
\hline $5 y$ & 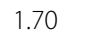 & 8 & 89.5 & 1.69 & 8 & $t$ & $2 \mathrm{cfh}$ & 4 & 63.8 & 66.7 & 1.53 & 84.2 & 5 & h & 6 & 2 & $B$ & & 6 & 58 \\
\hline 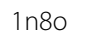 & & & 89.9 & & 82.9 & 7 & & 4 & & & & 83.0 & 84.1 & 0 & & 0 & 5 & & .4 & 0 \\
\hline uh & 1.98 & 8 & 70.3 & & 87.5 & 96.3 & & 4. & & 9 & & 0 & c & 1 & & .0 & 89.7 & & .9 & \\
\hline tt & 216 & 57.1 & 48.8 & 4.86 & 59.1 & 56.1 & n & 4 & 74.5 & 48.0 & 1.72 & 87.5 & & 13 & 7 & 7 & 72.2 & & 3 & 93 \\
\hline a9 & tha & 4 & 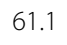 & 4.00 & 51.9 & 64.5 & 2 & 4 & 86.1 & 84.4 & 3.39 & 56.2 & 92.9 & r & 7 & 5 & 0 & & .0 & \\
\hline $\mathrm{Cq}$ & 2 & 90.9 & 75.3 & 5.19 & 7 & 6 & $1 \mathrm{ktz}$ & 4.84 & 80.0 & 69.6 & 3.68 & & 5 & $1 \mathrm{ibr}$ & 7.2 & 3 & 4 & & 6 & 33 \\
\hline $6 c$ & 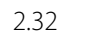 & 71.0 & 77.8 & 263 & 82.9 & 88.4 & 7 & 4 & 68.1 & 61.6 & 152 & 94.5 & 86.2 & 4 & 7 & 2 & 52.6 & & 85.7 & 90 \\
\hline 42 & 2.35 & 78.6 & 88.2 & & 94 & 87. & & 4.8 & & 66.7 & & & 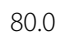 & b & 7. & 48.9 & 50 & & 3 & 72 \\
\hline $5 t$ & 2.38 & 8 & 7 & 4.36 & 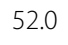 & 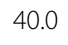 & 1jiw & 4.86 & 79.5 & 81.5 & 522 & 56.5 & 66.7 & $C$ & 7 & 3 & 44.4 & 1.47 & 2 & 97 \\
\hline 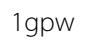 & 2 & 1 & 64.0 & 1.51 & 0 & 78.4 & $d$ & 4. & 73.3 & 02.5 & 3 & & 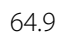 & b & & 1 & 64.8 & & J & 10 \\
\hline le & 2.47 & 78.6 & 73.2 & 0 & 74 & 44.0 & $1 \mathrm{f} 51$ & 4. & 70 & 686 & 2 & 66 & 68 & gl1 & 7. & 96.3 & 86.8 & & 2 & 83 \\
\hline do & 2.48 & & 82.8 & & & 80.0 & & & & & & & & 1 syx & & & & & & \\
\hline a) & 2.49 & 79 & 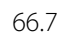 & 3.13 & 16 & P & $h$ & 4.9 & D & 79.0 & & 75.0 & 8 & leer & & .7 & 5 & & & 5 \\
\hline irk & 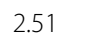 & 10 & 88.2 & 2.06 & 80 & 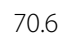 & 0 & 4 & 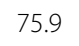 & 60.0 & 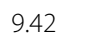 & 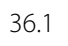 & 29.8 & $200 b$ & 7. & 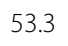 & 71.0 & 5.38 & 81.8 & \\
\hline qj & 2.52 & 79.2 & 91.3 & 8.3 & 53.7 & 35.9 & z & 4. & 54.8 & 30.0 & 3.73 & 89.7 & 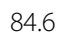 & 1jtg & 7. & 1 & 76.7 & & & \\
\hline :zu & 2.53 & 84 & 4.7 & 2.3 & 94 & 78.9 & $d$ & 5.0 & 81. & 77.1 & & 6 & 53 & 1nsn & 7. & .9 & 74.4 & & 1 & 8 \\
\hline 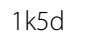 & 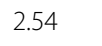 & 90.4 & 78.4 & 251 & 73.0 & 70.0 & 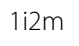 & 5 & 85.7 & 645 & & & 83.6 & 14 & 7 & 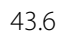 & 31.4 & & & \\
\hline ffw & 2.61 & 93.3 & 87.5 & 1.5 & 88.9 & 73. & e6j & 5.0 & 34.1 & 50.0 & 1. & 10 & 10 & 1udi & 8. & 51.1 & 50.0 & 1 & .9 & \\
\hline yo & 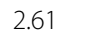 & 7 & 68.9 & 185 & 92.6 & 88.9 & 9 & 5. & 81.8 & 773 & 2 & 84.4 & 8 & $20 t 3$ & 8 & 3 & 71.6 & 4.40 & 2 & 73 \\
\hline nle & 2.63 & 55.6 & 58.8 & 3.52 & & & y & & 65.0 & & & & & h & & & & & & \\
\hline zhh & 2.67 & 66.7 & 70.4 & 9.28 & 27.5 & 45.6 & $1 \mathrm{kxp}$ & 5.13 & 62.0 & 78.8 & 2.00 & 80.0 & 66.7 & leaw & 8.31 & 95.2 & 85.3 & 1.34 & 92.9 & 92 \\
\hline 7 & 2.73 & 74.3 & 61.5 & 4.64 & 667 & 407 & $2 \mathrm{cOl}$ & 5.14 & 84.9 & 718 & & & 41.9 & q & 8 & . & 61.6 & & & \\
\hline $6 \mathrm{~m}$ & 2.76 & 84 & 8 & 12.24 & 26.1 & 19.2 & hi & 5 & 60.0 & 46.1 & & & c & n & & 7 & & & & \\
\hline $9 k$ & 2.80 & 89.7 & 81.0 & 5.67 & 62.1 & 37.8 & $1 p x v$ & 5.17 & 83.9 & 86.5 & 3.81 & 61.9 & 62.7 & $1 \mathrm{n} 2 \mathrm{c}$ & 8.66 & 86.4 & 78.9 & 1 & 5.7 & 92 \\
\hline 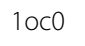 & 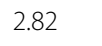 & & & 2.95 & 75.9 & & & 5 & 75.9 & & & & & & & & & & & \\
\hline $4 d$ & 2.97 & 71.1 & 65.3 & 1.9 & 68.4 & 64.9 & us7 & 5.1 & 55.0 & 76.2 & 1. & 88.0 & 84.6 & Trur & 0. & 6.5 & 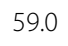 & & & 82 \\
\hline $8 v$ & 2.97 & 666 & 57.1 & 2.76 & 47 & 66 & 2jel & 5.2 & 0 & 73 & 2 & 3 & 79.1 & ej & 8.9 & 2.9 & 3 & 24.79 & 5.7 & 0 \\
\hline . & $\sqrt{5} .02$ & 1 & 70.2 & 1.54 & 94.6 & 87.5 & $1 \mathrm{fcc}$ & 5. & 66.7 & 41.0 & 11.33 & 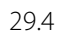 & 32.5 & q & S & 3 & 88.9 & & 0 & \\
\hline $\mathrm{mg} 8$ & 3. & 7 & 66.7 & 6.72 & 8 & 2 & & 5 & & 50.0 & 4.94 & 0 & 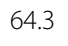 & b & 9 & 0 & 6 & 14.16 & 4 & \\
\hline 220 & 3.02 & 75.6 & 80.0 & 4.24 & 69.6 & 58.2 & $2 j 7 p$ & 5.38 & 66.7 & 64.7 & 6.89 & 50.9 & 59.0 & k & S & 9 & 86.2 & 15.2 & 21.7 & 2 \\
\hline nw9 & 3.02 & 70.6 & 66.7 & 3.19 & 78.8 & 68.6 & akj & 5.44 & 66.7 & 9.3 & 5.55 & 61.5 & 74.1 & $1 \mathrm{cgi}$ & 9.48 & 80.0 & 77.4 & 1.59 & 97.4 & 8 \\
\hline$f_{11}$ & 3.1 & 6 & 8 & 2.05 & 81.2 & $\beta$ & $1 \mathrm{ijk}$ & 5.46 & 60.6 & 44.5 & 1.86 & 7 & 3 & - & 9.48 & 1 & 6.6 & 1.58 & .9 & \\
\hline $9 r$ & 3.20 & 62.1 & 59.2 & 1.89 & 37.5 & 0 & רz8 & 5.49 & 2.8 & 75.0 & 2.87 & 82.6 & 5.8 & 7cei & 9.51 & 54.5 & 48.5 & 0.88 & 88.0 & \\
\hline
\end{tabular}


Table 14 The Docking Results of DoBi and ZDOCK on Benchmark v4.0 (Continued)

\begin{tabular}{|c|c|c|c|c|c|c|c|c|c|c|c|c|c|c|c|c|c|c|c|c|}
\hline 1 e96 & 3.21 & 96.5 & 84.6 & 2.98 & 55.2 & 63.2 & $1 \mathrm{~h} 9 \mathrm{~d}$ & 5.49 & 62.9 & 80.0 & 1.88 & 84.4 & 81.1 & 2vis & 9.57 & 81.0 & 91.4 & 22.23 & 0 & 0 \\
\hline$t 6 b$ & 3.22 & 71.2 & 70.2 & 1.19 & 85.0 & 90.9 & $1 \mathrm{rlb}$ & 5.50 & 76.2 & 58.8 & 14.71 & 26.7 & 23.8 & bgx & 9.90 & 8.3 & 80.0 & 11.09 & 50.5 & 27.2 \\
\hline oul & 3.30 & 64.9 & 65.5 & 1.97 & 80.0 & 86.8 & 1bj1 & 5.59 & 83.3 & 85.7 & 2.11 & 92.7 & 88.0 & $1 d 6 r$ & 10.54 & 41.0 & 66.6 & 12.68 & 25.0 & 22. \\
\hline . & 3.36 & 45.2 & 57.1 & 1.86 & 88.9 & 89.4 & $1 r 6 q$ & 5.59 & 50.0 & 78.6 & 5.20 & 47.4 & 53.3 & 2ajf & 10.60 & 52.4 & 51.1 & 3.57 & 2.3 & 69.6 \\
\hline 64 & 3.44 & 94.1 & 88.9 & 15.37 & 31.6 & 21.6 & $1 \mathrm{gfw}$ & 5.61 & 48.0 & 57.2 & 1.50 & 93.3 & 77.8 & $1 \mathrm{ml} 0$ & 10.69 & 54.0 & 42.4 & 1.29 & 2.6 & 00 \\
\hline fw & 3.44 & 42.1 & 66.7 & 3.91 & 56.0 & 57.1 & 2uuy & 5.66 & 66.7 & 62.3 & 4.20 & 44.5 & 76.2 & $1 \mathrm{k} 74$ & 10.73 & 39.1 & 20.2 & 1.63 & 76.6 & 80.8 \\
\hline 1 rrn & 3.46 & 78.8 & 70.3 & 1.81 & 69.4 & 70.0 & 1 iqd & 5.66 & 64.9 & 70.4 & 1.26 & 94.4 & 80.0 & $1 \mathrm{dfj}$ & 11.14 & 48.3 & 35.5 & 1.29 & 77.9 & 82 \\
\hline $\mathrm{xcc}$ & 3.52 & 65.4 & 66.7 & 5.34 & 76.5 & 43.3 & 2 oza & 5.71 & 60.5 & 69.2 & 8.49 & 40.8 & 28.9 & $1 \mathrm{kac}$ & 11.24 & 74.1 & 42.9 & 3.22 & 87.8 & 8 \\
\hline 1hcf & 3.57 & 75.9 & 71.4 & 0.95 & 90.9 & 86.5 & 1 fak & 5.73 & 71.4 & 86.3 & 7.73 & 40.0 & 44.9 & $1 \times 41$ & 11.36 & 87.5 & 78.8 & 1.54 & 39.7 & \\
\hline $1 \mathrm{a} 2 \mathrm{k}$ & 3.57 & 75.7 & 50.0 & 1.91 & 55.8 & 53.7 & $1 \mathrm{de} 4$ & 5.76 & 53.9 & 70.0 & 1.77 & 80.0 & 78.4 & $1 \mathrm{mah}$ & 11.55 & 86.9 & 73.5 & 1.87 & 86.5 & 83. \\
\hline wh & 3.61 & 66.7 & 75.6 & 1.28 & 80.0 & 66.7 & 1zgi & 5.82 & 90.9 & 88.2 & 1.79 & 78.3 & 85.7 & 1he8 & 11.95 & 58.3 & 56.3 & 2.38 & 60.0 & \\
\hline atn & 3.70 & 72.3 & 83.3 & 4.74 & 79.1 & 80.0 & 1azs & 5.86 & 62.9 & 75.9 & 1.18 & 84.2 & 83.3 & $1 \mathrm{fsk}$ & 11.99 & 61.1 & 62.8 & 1.15 & 91.9 & . \\
\hline ic & 3.76 & 72.2 & 76.4 & 0.94 & 96.3 & 90.9 & 1 hia & 5.91 & 66.7 & 56.1 & 12.4 & 23.0 & 28.6 & $1 \mathrm{~h} 1 \mathrm{v}$ & 14.13 & 20.4 & 38.9 & 16.72 & 18.2 & 20. \\
\hline 1ppe & 3.83 & 76.9 & 83.3 & 1.42 & 86.7 & 93.5 & $1 \mathrm{mlc}$ & 6.1 & 54.2 & 71.9 & 1.5 & 80.0 & 78.9 & $1 \times q s$ & 14.27 & 37.2 & 23.3 & 1.67 & 79.1 & \\
\hline $1 \mathrm{klu}$ & 3.94 & 83.7 & 90.9 & 11.1 & 27.5 & 43.2 & $2 \mathrm{fd} 6$ & 6.20 & 63.0 & 53.3 & 4.34 & 75.9 & 43.4 & $1 \mathrm{jk} 9$ & 15.43 & 84.4 & 74.4 & 2.16 & 82.9 & \\
\hline 17 & 3.97 & 87.1 & 71.4 & 12.25 & 43.2 & 28.6 & 4сра & 6.21 & 62.1 & 0 & 1.7 & 80.0 & 81.0 & 1ghq & 16.12 & 68.3 & 57.7 & 22.15 & 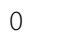 & \\
\hline $\mathrm{d} 5 \mathrm{~s}$ & 3.97 & 70.0 & 72.3 & 2.08 & 81.1 & 84.2 & 1nca & 6.25 & 64.5 & 71.0 & 1.38 & 90.2 & 87.0 & $1 \mathrm{r} 8 \mathrm{~s}$ & 20.83 & 12.6 & 57.7 & 6.48 & 49.2 & \\
\hline $2 \mathrm{~b} 4 \mathrm{i}$ & 4.00 & 82.4 & 83.7 & 10.33 & 35.7 & 28.6 & $1 \mathrm{f} 34$ & 6.36 & 59.3 & 61.5 & 1.94 & 84.8 & 83.6 & $1 \mathrm{kxq}$ & 21.12 & 75.0 & 80.0 & 1.18 & 84.6 & \\
\hline i9b & 4.18 & 80.0 & 79.2 & 5.58 & 78.3 & 42.1 & 1ib1 & 6.42 & 64.8 & 71.4 & 5.89 & 53.1 & 46.4 & $2 \mathrm{hqs}$ & 26.33 & 10.5 & 22.6 & 12.37 & 15.0 & . \\
\hline $2 \mathrm{mta}$ & 4.18 & 82.1 & 86.5 & 1.64 & 84.6 & 82.4 & 3bp8 & 6.49 & 61.0 & 63.2 & 4.02 & 57.9 & 68.8 & 1 ira & 28.13 & 31.8 & 25.0 & 16.42 & 36.5 & . \\
\hline b7 & 4.19 & 72.2 & 81.2 & 2.64 & 85.7 & 80.0 & & & & & & & & & & & & & & \\
\hline
\end{tabular}

${ }^{a} C_{\alpha}$ iRMSD between the configuration by methods and the experimental structure.

${ }^{b} F_{/}(\%)$ is the F-score of each method for the ligand protein on the data set.

${ }^{c} F_{r}(\%)$ is the $\mathrm{F}$-score of each method for the receptor protein on the data set.

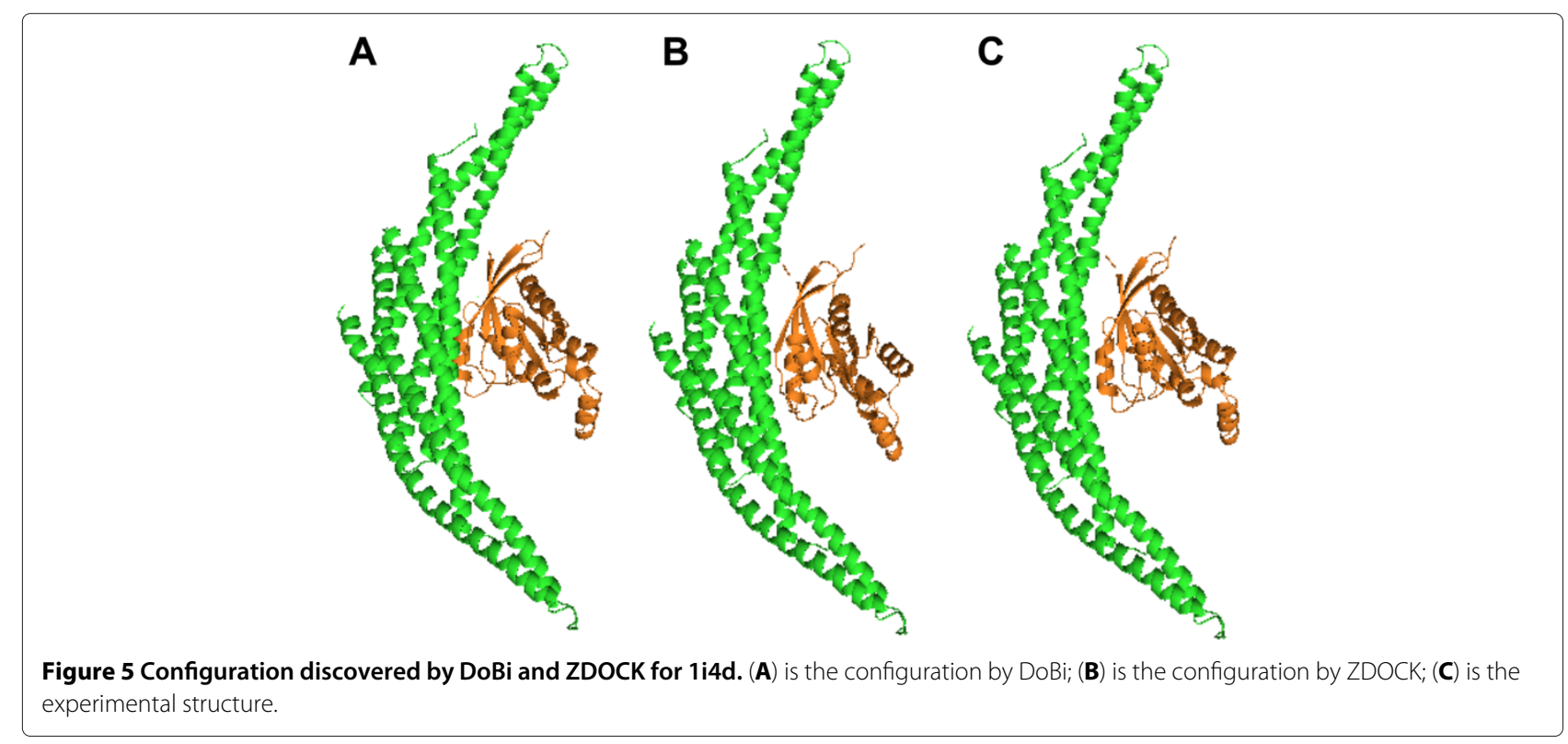


cases. The success rate of $\mathrm{DoBi}$ is $41.7 \%$ for the rigid-body cases, which is significantly better than for the other two categories. In general, the accuracy and coverage decrease as the magnitude of conformational increases. The details are shown in Table 12.

DoBi discovered several good configurations for the medium difficult cases. One of the instances is $1 \mathrm{wq} 1(\mathrm{R}: \mathrm{G})$. Its configuration discovered by DoBi is shown in Figure 4. The $C_{\alpha}$ iRMSD between the experimental structure and the predicted complex is $4.12 \AA$.

\section{Docking result of DoBi}

$\mathrm{DoBi}$ is optimized for binding site prediction, but it also can be used to dock two protein structures. We compare DoBi's poses to the best configurations obtained by ZDOCK and 3D-Dock. ZDOCK [35] uses a fast Fourier transform to search all possible binding modes for the proteins, and evaluates them based on shape complementarity, desolvation energy, and electrostatics. It can produce structures with the smallest iRMSD values in top 1000 predictions with minimum energy. 3D-Dock[36,37] uses an initial grid-based shape complementarity search to produce lots of potential interacting conformations. They can be ranked by using interface residue propensities and interaction energies. It reports structures with the smallest iRMSD values in top ten predictions.

We calculate the predicted structures by different methods on the complexes in benchmark v4.0 and the targets in CAPRI. CAPRI is a community-wide experiment to assess the capacity of protein docking methods to predict protein-protein interactions [31]. The $C_{\alpha}$ iRMSD, F-score and the fraction of native contacts are used to evaluate the results by different methods. The fraction of native contacts is used by 3D-Dock[37]. It is calculated as the total number of native contacts for the predicted configuration divided by the total number of contacts in the native structure. A native contact exists between residues $i$ and $j$ if distances between them in native structure and in predicted configuration are both less than $4.5 \AA$.

We compare the docking results of DoBi, ZDOCK and 3D-DOCK on the CAPRI targets. The results are shown in Table 13. The top 1,000 configurations predicted by DoBi and ZDOCK are used for comparison. Among the 1,000 predictions, we choose the configuration of the best iRMSD value to evaluate the methods. The average iRMSD values for DoBi and ZDOCK are $7.5 \AA$ and $6.9 \AA$, respectively. However, the average fractions of native contacts for $\mathrm{DoBi}$ and ZDOCK are $40.6 \%$ and $35.2 \%$, respectively. DoBi improves the F-score of binding site prediction by at least $1.3 \%$. DoBi's performance on docking is worse than ZDOCK, but its performance on binding site prediction is more accurate than ZDOCK.
Each of DoBi and 3D-Dock produced ten results for each target, and the configurations with smallest iRMSD values among those ten predictions are used for comparison. The average iRMSD values for DoBi and 3D-Dock are $9.2 \AA$ and $9.1 \AA$. However, the overall fractions of native contacts for DoBi and 3D-Dock are $29.1 \%$ and $26.8 \%$. DoBi's performance on binding site prediction is better than that of 3D-Dock.

The docking results obtained by DoBi and ZDOCK on Benchmark v4.0 are shown in Table 14. Similarly, we compare the best configurations in the top 1000 predictions from each method of DoBi and ZDOCK for each target. The average iRMSD values of DoBi and ZDOCK are $6.1 \AA$ and $4.9 \AA$, respectively. For the binding site prediction, the overall $\mathrm{F}$-score values of ligand proteins by DoBi and ZDOCK are $69.5 \%$ and $69.4 \%$, and those of receptor proteins by DoBi and ZDOCK are $68.2 \%$ and $66.1 \%$, respectively. These results indicate that DoBi's performance on binding site prediction is better than ZDOCK. The docking quality of DoBi requires further efforts to improve.

We calculate the docking results of $1 \mathrm{i} 4 \mathrm{~d}$. The $C_{\alpha}$ iRMSD values between the experimental structure and the configurations by DoBi and ZDOCK are $2.97 \AA$ and $1.97 \AA$, respectively. DoBi improves F-score value of ligand protein by $2.7 \%$, and that of receptor protein by $0.4 \%$. The configurations produced by methods are shown in Figure 5.

\section{Factors affecting the performance of DoBi}

We notice that DoBi performed badly on a few specific instances. We analyze this performance issue with Table 15, which compares the ACE scores for the experimental structures and predicted complexes, for the bound states of proteins in the benchmark v4.0. Among the 176 complexes, only 43 of them have an ACE score for experimental structures lower than that of the predicted complexes. This implies that in 133 cases, DoBi is able to find a configuration of a lower score than the experimental structures. These anomalies suggest that the score function currently used in DoBi may be inaccurate, and this inaccuracy may have contributed to the poorly performed cases of DoBi. We also note that the search space currently explored by our method is incomplete, and this may have contributed as well to the inaccuracy of DoBi in some cases.

Figure 6 shows the protein complex incorrectly predicted by DoBi as well as the experimental structure for $1 \mathrm{kxq}(\mathrm{H}: \mathrm{A})$. The iRMSD between the two complexes is $18.87 \AA$. The ACE score of the docking structure predicted by $\mathrm{DoBi},-497.6$, is lower than the ACE score of the experimental structure, 63.7. The binding sites predicted by DoBi are incorrect as well. 
Table 15 Comparison of Atomic Contact Energy for the Predicted Complexes and the Experimental Structures on Benchmark v4.0

\begin{tabular}{|c|c|c|c|c|c|c|c|c|c|c|c|c|c|c|c|c|c|c|c|}
\hline PDB & $\mathrm{E}_{\text {act }^{\mathrm{a}}}$ & $\mathrm{E}_{p r e}{ }^{\mathrm{b}}$ & $\mathrm{F}_{r}^{\mathrm{c}}$ & $\mathrm{F}_{l}^{\mathrm{d}}$ & PDB & $\mathrm{E}_{\text {act }}$ & $\mathrm{E}_{\text {pre }}$ & $\mathrm{F}_{r}$ & $\mathrm{~F}_{l}$ & PDB & $\mathrm{E}_{\text {act }}$ & $\mathrm{E}_{\text {pre }}$ & $\mathrm{F}_{r}$ & $\mathrm{~F}_{l}$ & PDB & $\mathrm{E}_{\text {act }}$ & $\mathrm{E}_{\text {pre }}$ & $\mathrm{F}_{r}$ & $\mathrm{~F}_{l}$ \\
\hline $208 v(A: B)$ & -96.7 & -149.3 & 91.4 & 81.0 & $1 \mathrm{a} 2 \mathrm{k}(\mathrm{C}: \mathrm{B})$ & -38.9 & -314.8 & 72.7 & 71.8 & $2 \operatorname{vis}(B: C)$ & 35.8 & -389.7 & 62.8 & 61.1 & $1 n 2 c(A: F)$ & 97.6 & -272.1 & 46.1 & 60.0 \\
\hline $1 \mathrm{hcf}(\mathrm{A}: \mathrm{X})$ & -46.2 & -13.4 & 90.9 & 83.7 & 4cpa(A:I) & -47.6 & -318.3 & 72.7 & 71.0 & $1 \mathrm{acb}(\mathrm{E}: \mathrm{I})$ & -157.4 & -555.7 & 62.2 & 92.9 & $1 \times u 1(A: T)$ & 60.7 & 85.9 & 45.5 & 72.7 \\
\hline $1 z 5 y(D: E)$ & -85.9 & -51.1 & 89.7 & 90.0 & 1 wq1 (R:G) & 161.0 & 306.0 & 72.4 & 77.3 & 1j2j(A:B) & -49.1 & -161.8 & 62.1 & 51.6 & $1 g p w(A: B)$ & 107.8 & -144.5 & 45.0 & 75.7 \\
\hline $\operatorname{lgcq}(B: C)$ & -5.3 & 4.4 & 88.9 & 94.1 & $1 \mathrm{mah}(\mathrm{A}: \mathrm{F})$ & -8.5 & -303.0 & 72.4 & 64.3 & $1 \mathrm{jzd}(\mathrm{A}: C)$ & 55.2 & 78.5 & 61.6 & 57.1 & $10 y v(B: I)$ & -70.5 & -136.0 & 44.5 & 60.6 \\
\hline 2jot(A:D) & -65.8 & -74.5 & 88.9 & 93.3 & 1udi(E:I) & 69.2 & -93.0 & 72.0 & 50.0 & $2 \mathrm{hmi}(\mathrm{D}: \mathrm{B})$ & -7.1 & -524.8 & 61.6 & 57.2 & 1d6r(A:I) & 88.0 & -66.6 & 43.4 & 66.7 \\
\hline $1 \mathrm{~s} 1 \mathrm{q}(\mathrm{A}: \mathrm{B})$ & 20.4 & 168.1 & 88.2 & 90.9 & $1 \mathrm{t} 6 \mathrm{~b}(X: Y)$ & 87.4 & -675.9 & 71.9 & 54.2 & $1 \mathrm{vfb}(\mathrm{B}: \mathrm{C})$ & 81.7 & 191.4 & 61.5 & 59.3 & $2 f j u(B: A)$ & 61.0 & -658.9 & 43.1 & 27.9 \\
\hline 2ayo(A:B) & 340.9 & 384.2 & 86.8 & 96.3 & $2 g 77(A: B)$ & 215.5 & 72.6 & 71.6 & 76.3 & $1 b 6 c(A: B)$ & -29.8 & -130.3 & 61.1 & 47.6 & $2 \operatorname{sic}(E: I)$ & -158.8 & -321.2 & 42.9 & 74.1 \\
\hline 1n8o(C:E) & -91.8 & -64.1 & 86.5 & 83.9 & $1 w d w(B: A)$ & 301.6 & 30.6 & 71.4 & 64.8 & $10 p h(A: B)$ & -10.1 & -516.1 & 60.6 & 64.5 & 1eer(A:B) & 143.2 & 219.2 & 42.1 & 71.4 \\
\hline $\mathrm{d}(\mathrm{D}: \mathrm{A})$ & 30.0 & -49.1 & 86.5 & 82.1 & 1jk9(B:A) & 1.7 & -150 & 71 & 69 & $1 f$ & 17 & 37.4 & 60.0 & 47.4 & 1 & -32.7 & $-1 c$ & 41.8 & 62.9 \\
\hline 1qa9(A:B) & 260.0 & -73.9 & 85.7 & 83.3 & 1gp2(A:B) & 56.5 & -48.6 & 71.4 & 72.7 & $2 \mathrm{i} 25(\mathrm{~N}: \mathrm{L})$ & 131.0 & 144.8 & 60.0 & 76.6 & 2abz(B:E) & 34.0 & -300.7 & 41.0 & 70.3 \\
\hline 2hle(A:B) & 83.0 & 179.3 & 85.3 & 95.2 & 1dqj(B:C) & 119.4 & 104.1 & 71.4 & 71.4 & $1 \mathrm{ktz}(\mathrm{A}: \mathrm{B})$ & -24.0 & -150.3 & 60.0 & 57.2 & $1 \mathrm{fq} 1(\mathrm{~A}: \mathrm{B})$ & 152.5 & -187.8 & 40.8 & 30.8 \\
\hline 1 fle(E:I) & -134.1 & -248.0 & 84.6 & 96.5 & 1 us $7(A: B)$ & 71.6 & 30.8 & 71.0 & 64.5 & $1 \mathrm{i} 9 \mathrm{r}(\mathrm{H}: \mathrm{A})$ & 92.9 & 284.5 & 60.0 & 58.8 & 1.jps(H:T) & 258.6 & 366.2 & 37.8 & 66.7 \\
\hline 1jtg (B:A) & 232.8 & 257.6 & 84.4 & 86.1 & $1 \mathrm{kkl}(\mathrm{A}: \mathrm{H})$ & 105.2 & -252 & 71.0 & 75.0 & $1 \mathrm{sbb}(\mathrm{A}: \mathrm{B})$ & 05 & 154.1 & 60.0 & 58.8 & $1 \times q s(A: C)$ & 368.9 & 383.0 & 37.2 & 23.3 \\
\hline 1hia(B:I) & -4.8 & 51.3 & 83.7 & 56.0 & $3 d 5 s(A: C)$ & 89.8 & -70.8 & 70.8 & 68.3 & :B) & 79.2 & 68.9 & 59.2 & 62.1 & $1 z m 4(A: B)$ & 118.6 & -236.7 & 35.8 & 33.3 \\
\hline $1 \mathrm{k} 5 \mathrm{~d}(\mathrm{~A}: \mathrm{C})$ & 197.6 & 305.1 & 81.5 & 79.5 & $1 \mathrm{r} 6 \mathrm{q}(\mathrm{A}: \mathrm{C})$ & -71.5 & -129.3 & 70.4 & 64.9 & $2 \mathrm{igb}(\mathrm{E}: \mathrm{A})$ & 58.0 & -87.2 & 59.0 & 76.5 & $1 \mathrm{ijk}(\mathrm{C}: \mathrm{A})$ & 85.1 & -45.5 & 35.7 & 14.8 \\
\hline 1yvb(A:l) & -141.9 & -271.2 & 80.8 & 72.2 & 1he1(C:A) & 20.6 & 242.2 & 70.3 & 66.6 & $1 p x v(A: C)$ & 28.5 & -79.4 & 58.8 & 76.2 & 1 tmq(A:B) & 2.1 & -466.6 & 35.1 & 63.0 \\
\hline $1 \mathrm{fak}(\mathrm{L}: T)$ & 108.7 & 217.2 & 80.0 & 75.6 & 1rv6(V:X) & -17.7 & -3.7 & 70.0 & 52.6 & 1ror(E:I) & -126.0 & -127.4 & 58.8 & 61.5 & 2ido(A:B) & -71.8 & 92.8 & 33.4 & 39.0 \\
\hline $3 s g q(E: I)$ & -57.9 & 26.3 & 80.0 & 75.0 & $1 \mathrm{bkd}(\mathrm{R}: \mathrm{S})$ & 195.0 & -49.0 & 69.6 & 78.6 & 1e6j(H:P) & 14.9 & -366.7 & 58.8 & 40.0 & $10 c 0(A: B)$ & 27.1 & -417.1 & 33.3 & 66.7 \\
\hline $1 \mathrm{pvh}(\mathrm{A}: \mathrm{B})$ & 121.5 & 13.5 & 80.0 & 62.9 & $1 \mathrm{avx}(\mathrm{A}: \mathrm{B})$ & 31.8 & 35.1 & 69.2 & 81.5 & 1jmo(A:H) & -49.0 & -492.6 & 57.9 & 32.5 & $1 y 64(A: B)$ & 123.8 & -239.0 & 32.3 & 36.0 \\
\hline $200 b(A: B)$ & -15.8 & -28.9 & 80.0 & 78.3 & 1zhi(A:B) & 93.8 & -89.3 & 68.7 & 64.5 & $3 \operatorname{cph}(G: A)$ & 84.4 & -193.3 & 57.7 & 68.3 & $1 \mathrm{dfj}(\mathrm{E}: \mathrm{l})$ & 159.3 & -394.3 & 32.1 & 44.4 \\
\hline 1oyv(A:I) & -152.8 & -158.1 & 79.3 & 66.7 & $1 \mathrm{kac}(\mathrm{A}: \mathrm{B})$ & 92.6 & 66.9 & 68.6 & 57.9 & $1 \mathrm{ewy}(\mathrm{A}: \mathrm{C})$ & 55.6 & -80.1 & 57.2 & 63.1 & $10(A: B)$ & 168.3 & -36.2 & 31.8 & 54.1 \\
\hline $1 \mathrm{i} 2 \mathrm{~m}(\mathrm{~A}: \mathrm{B})$ & 300.9 & 213.4 & 79.2 & 79.4 & $\lg \mid 1(\mathrm{~A}: \mathrm{l})$ & -83.2 & -282.7 & 68.1 & 78.8 & $2 \mathrm{~h} 7 \mathrm{~V}(\mathrm{~A}: \mathrm{C})$ & 67.5 & 9.9 & 57.2 & 73.2 & 1ira(Y:X) & 212.7 & 48.1 & 31.8 & 25.0 \\
\hline 1atn(A:D) & -72.3 & -365.5 & 79.1 & 73.3 & 1 e6e(A:B) & 246.8 & -137.5 & 67.7 & 73.4 & $1 \mathrm{gfw}(\mathrm{M}: \mathrm{B})$ & 60.9 & 61.7 & 57.2 & 48.0 & $20 u l(A: B)$ & -123.9 & -311.2 & 30.4 & 54.0 \\
\hline $1 \mathrm{klu}(\mathrm{A}: \mathrm{D})$ & 60.2 & -243.4 & 79.0 & 54.5 & $1 \mathrm{bj} 1(\mathrm{H}: \mathrm{W})$ & 10.9 & -139.6 & 66.7 & 71.0 & $2 z 0 e(A: B)$ & -38.7 & -562.7 & 56.4 & 63.8 & $1 \mathrm{k} 74(\mathrm{~A}: \mathrm{D})$ & 127.4 & 145.5 & 30.0 & 27.3 \\
\hline (A:B) & -5.4 & -52.5 & 78.9 & 86.4 & $1 \mathrm{k} 4 \mathrm{C}(\mathrm{A}: \mathrm{C})$ & 70.3 & -216.1 & 66.7 & 52.2 & $2 \mathrm{vdb}(\mathrm{A}: \mathrm{B})$ & 77.4 & -562.9 & 56.3 & 58.3 & $(A: B)$ & -0.5 & -175.5 & 30.0 & 42.1 \\
\hline 1 efn(B:A) & 30.0 & 173.4 & 78.8 & 89.7 & $1 \mathrm{fc} 2(\mathrm{C}: \mathrm{D})$ & 23.1 & -93.1 & 66.7 & 70.6 & $1 f 6 m(A: C)$ & 14.6 & -307.7 & 56.0 & 63.8 & 3bp8(A:C) & 57.9 & -429.9 & 30.0 & 53.0 \\
\hline 1 buh(A:B) & 70.5 & 151.9 & 78.8 & 71.1 & 2jel(H:P) & 74.0 & 18.2 & 66.7 & 75.9 & $1 \mathrm{e} 4 \mathrm{k}(\mathrm{A}: \mathrm{C})$ & -41.5 & -385.7 & 55.1 & 48.0 & 1azs(A:C) & -65.7 & -331.5 & 28.6 & 51.4 \\
\hline 2sni(E:I) & -125.0 & -1.9 & 78.8 & 87.5 & 1zhh(A:B) & -84.0 & -537.9 & 66.7 & 64.3 & 1zli(A:B) & -100.2 & -164.6 & 54.1 & 52.4 & 1he8(B:A) & 64.0 & -323.3 & 27.4 & 40.0 \\
\hline $1 \mathrm{mlc}(\mathrm{B}: \mathrm{E})$ & 74.4 & -133.7 & 78.8 & 62.0 & $1 \mathrm{gla}(\mathrm{G}: \mathrm{F})$ & -26.3 & -232.4 & 66.7 & 65.4 & $1 \mathrm{kxp}(\mathrm{A}: D)$ & 189.4 & -311.2 & 54.0 & 54.8 & $2 f d 6(H: U)$ & 78.6 & -317.1 & 27.3 & 31.6 \\
\hline $1 \mathrm{gfw}(\mathrm{H}: \mathrm{A})$ & 36.5 & 150.4 & 78.6 & 45.4 & $1 \mathrm{mlO}(\mathrm{A}: \mathrm{D})$ & -95.8 & -641.1 & 66.7 & 60.4 & $1 \mathrm{clv}(\mathrm{A}: \mathrm{I})$ & 0.3 & -648.0 & 54.0 & 79.1 & 1 fqj(A:B) & 234.8 & 326.9 & 26.4 & 25.9 \\
\hline $1 \times d 3(A: B)$ & -5.2 & -240.3 & 78.0 & 63.4 & $1 z 0 k(A: B)$ & 9.7 & -84.1 & 66.6 & 80.0 & $1 \operatorname{de} 4(A: C)$ & 123.1 & -535.6 & 54.0 & 49.3 & $1 \operatorname{syx}(A: B)$ & 116.4 & 113.1 & 26.3 & 66.7 \\
\hline
\end{tabular}


Table 15 Comparison of Atomic Contact Energy for the Predicted Complexes and the Experimental Structures on Benchmark v4.0 Continued

\begin{tabular}{|c|c|c|c|c|c|c|c|c|c|c|c|c|c|c|c|c|c|c|c|}
\hline $2 \mathrm{mta}(\mathrm{L}: \mathrm{A})$ & -55.7 & 70.5 & 77.4 & 80.0 & 2nz8(A:B) & 52.1 & 36.3 & 66.6 & 77.1 & 1jiw(P:I) & 110.3 & -628.9 & 53.9 & 66.7 & $2 b 42(A: B)$ & 103.6 & -199.4 & 24.0 & 23.6 \\
\hline 1nw9(B:A) & -120.1 & -333.9 & 77.3 & 80.0 & $1 e 96(A: B)$ & 110.7 & -120.4 & 66.6 & 60.6 & $2 b 4 j(A: C)$ & 94.0 & -120.6 & 53.8 & 66.7 & 1eaw(A:B) & 12.0 & -173.1 & 23.2 & 74.3 \\
\hline 2c0l(A:B) & 130.2 & -225.3 & 76.7 & 78.1 & 2oor(A:C) & -50.4 & -839.7 & 66.6 & 41.0 & 1ezu(C:B) & -103.2 & -172.2 & 53.1 & 66.7 & $2 p c c(A: B)$ & 47.3 & 98.9 & 22.2 & 30.3 \\
\hline $1 \operatorname{iqd}(A: C)$ & -14.3 & -261.7 & 76.5 & 52.2 & 2ajf(A:E) & 59.4 & -194.7 & 64.8 & 57.1 & 1rlb(B:E) & -69.1 & -322.3 & 52.7 & 63.2 & $\operatorname{lgxd}(A: C)$ & 45.2 & -680.4 & 21.9 & 72.1 \\
\hline 1nsn(L:S) & 73.6 & 122.8 & 76.2 & 38.7 & 1ahw(B:C) & 262.7 & 388.1 & 64.7 & 80.0 & $1 \mathrm{ibr}(\mathrm{A}: \mathrm{B})$ & 234.0 & -850.1 & 51.3 & 38.5 & $2 j 7 p(A: D)$ & 208.9 & 122.5 & 21.7 & 30.2 \\
\hline 1nca(H:N) & 146.6 & 78.6 & 75.9 & 66.6 & $1 \mathrm{lfd}(\mathrm{B}: \mathrm{A})$ & 85.3 & -28.0 & 64.5 & 85.7 & $20 t 3(B: A)$ & -165.8 & -494.1 & 51.1 & 52.4 & $1 \mathrm{~h} 1 \mathrm{v}(\mathrm{A}: \mathrm{G})$ & 115.0 & -60.2 & 20.4 & 38.9 \\
\hline 2z9k(A:B) & 67.0 & -89.9 & 75.6 & 83.3 & 1 ay7(A:B) & 123.2 & -30.3 & 64.5 & 77.4 & 1cgi(E:I) & -186.4 & -383.8 & 51.0 & 80.0 & 2oza(B:A) & 287.3 & -5.2 & 20.2 & 39.1 \\
\hline $\operatorname{lgrn}(A: B)$ & 189.3 & -80.6 & 75.6 & 66.7 & $2 b t f(A: P)$ & 165.6 & 102.3 & 64.0 & 50.0 & 1akj(A:D) & 108.3 & 11.1 & 51.0 & 61.6 & $1 \mathrm{kxq}(\mathrm{H}: \mathrm{A})$ & 63.7 & -497.6 & 19.7 & 28.1 \\
\hline 1 bgx $(L: T)$ & 127.3 & -727.9 & 75.3 & 59.5 & $1 \mathrm{bvk}(\mathrm{E}: \mathrm{F})$ & 76.8 & 150.6 & 64.0 & 46.1 & $1 \mathrm{f3} 4(\mathrm{~A}: \mathrm{B})$ & -70.5 & -376.9 & 49.3 & 66.6 & 1jwh(C:A) & -27.8 & -305.7 & 18.7 & 35.3 \\
\hline 1ppe(E:I) & -54.5 & -6.3 & 75.0 & 72.8 & $1 \mathrm{~h} 9 \mathrm{~d}(\mathrm{~A}: \mathrm{B})$ & 12.9 & 167.7 & 63.6 & 72.4 & $1 \mathrm{fsk}(\mathrm{C}: \mathrm{A})$ & 60.7 & -19.8 & 48.3 & 45.2 & $1 \mathrm{bvn}(\mathrm{P}: \mathrm{T})$ & -43.9 & -785.8 & 18.5 & 65.1 \\
\hline $2 \mathrm{cfh}(\mathrm{A}: \mathrm{C})$ & -162.0 & -435.9 & 74.4 & 73.9 & 7cei(A:B) & 216.5 & 192.5 & 63.2 & 68.8 & 1ak4(A:D) & -48.6 & 60.8 & 47.1 & 56.0 & $203 b(A: B)$ & 119.0 & -17.4 & 14.3 & 28.6 \\
\hline $1 \mathrm{fcc}(\mathrm{A}: \mathrm{C})$ & 247.3 & 160.9 & 74.1 & 66.7 & 1 wej(L:F) & 117.5 & 48.0 & 63.2 & 50.0 & $1 \mathrm{mq} 8(\mathrm{~A}: \mathrm{B})$ & 40.7 & -56.3 & 46.7 & 84.9 & $1 \mathrm{r} 8 \mathrm{~s}(\mathrm{~A}: \mathrm{E})$ & 38.2 & 90.0 & 12.6 & 57.7 \\
\hline 2uuy(A:B) & -10.2 & -127.7 & 73.5 & 86.9 & 1ib1(A:E) & 163.1 & 240.4 & 62.8 & 63.4 & $2 a 5 t(A: B)$ & 107.0 & -227.5 & 46.5 & 48.9 & 2hqs(A:H) & 190.9 & -202.6 & 10.5 & 22.6 \\
\hline
\end{tabular}

${ }^{\mathrm{a}} \mathrm{E}_{\text {act }}$ is $\mathrm{ACE}$ score for the experimental structure on the data set.

${ }^{\mathrm{b}} \mathrm{E}_{\text {pre }}$ is ACE score for the prediction complex on the data set.

${ }^{c} F_{r}(\%)$ is the F-score of our method for the receptor protein on the data set.

${ }^{d} F_{/}(\%)$ is the F-score of our method for the ligand protein on the data set. 

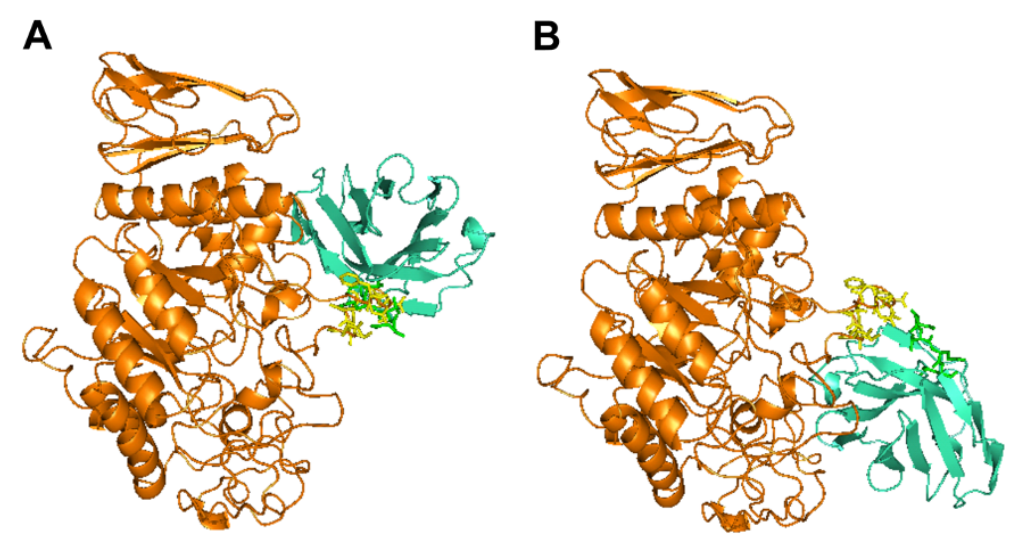

Figure 6 DoBi fails to solve the instance $\mathbf{1} \mathbf{k x q}(\mathbf{H : A}) .(\mathbf{A})$ is the predicted complex; and $(\mathbf{B})$ is the experimental structure.

\section{Conclusions}

In this work, we proposed an approach to identify binding sites in protein complexes by docking protein subunits. The method is implemented in a program called DoBi. DoBi consistently and significantly performed better than existing techniques in predicting binding sites in experimental results.

We identify a few potential areas for future improvements to our method. The first area to work on is in the energy function used. Currently, DoBi uses a simple score function. As suggested by the experiment results, a better energy function is able to improve the performance of DoBi.

A second area for improvement is in our current assumption that protein structures are rigid when binding. In reality, protein structures may vary sightly or even dramatically when they bind. Hence, further studies on this issue are very much in demand.

Although our method shows better overall performance, there are some protein complexes where other methods outperformed DoBi. It will be beneficial if we could combine the strengths of these existing programs with $\mathrm{DoBi}$, to come up with a more reliable method.

\section{Endnote}

${ }^{a}$ The initial two letters from each of the two words, Docking and Binding, were taken.

\section{Competing interests}

The authors declare that they have no competing interests.

\section{Acknowledgements}

This work is supported by a grant from the Research Grants Council of the Hong Kong Special Administrative Region, China [Project No. CityU 122511] and a grant from City University of Hong Kong [Project No. 9610025].

\section{Author details}

${ }^{1}$ School of Computer Science and Technology, Shandong University, Jinan 250101, Shandong, China. ${ }^{2}$ Department of Computer Science, City University of Hong Kong, 83 Tat Chee Avenue, Kowloon, Hong Kong.

\section{Authors' contributions}

FG participated in the design of the study, performed the statistical analysis, and is in charge of the software package development. SL participated in the experiment design and drafted the manuscript. LW conceived of the study, participated in its design, and helped to draft the manuscript. DZ is heavily involved in the computation of the tables. All authors read and approved the final manuscript.

Received: 8 December 2011 Accepted: 15 June 2012

Published: 6 July 2012

\section{References}

1. Chung $\mathrm{L}$, , Wang W, Bourne PE: Exploiting sequence and structure homologs to identify protein-protein binding sites. Proteins 2006, 62:630-640.

2. Ofran $Y$, Rost B: Protein-protein interaction hotspots carved into sequences. PLoS Comput Biol 2007, 3(7):1169-1176.

3. Šikić M, Tomić S, Vlahoviček K: Prediction of Protein-protein interaction sites in sequences and 3D structures by random forests. PLoS Comput Biol 2009, 5(1):1-9.

4. Chen $X$, Jeong JC: Sequence-based prediction of protein interaction sites with an integrative method. Bioinformatics 2009, 25(5):585-591.

5. Murakami Y, Mizuguchi K: Applying the Naïve Bayes classifier with kernel density estimation to the prediction of protein-protein interaction sites. Bioinformatics 2010, 26(15):1841-1848.

6. Konc J, Janežič D: ProBiS algorithm for detection of structurally similar protein binding sites by local structural alignment. Bioinformatics 2010, 26(9):1160-1168.

7. Burgoyne NJ, Jackson RM: Predicting protein interaction sites: binding hot-spots in protein-protein and protein-ligand interfaces. Bioinformatics 2006, 22(11):1335-1342.

8. Ortuso F, Langer T, Alcaro S: GBPM: GRID-based pharmacophore model: concept and application studies to protein-protein recognition. Bioinformatics 2006, 22(12):1449-1455.

9. Neuvirth $H, R a z R$, Schreiber G: ProMate: a structure based prediction program to identify the location of protein-protein binding sites. J Mol Biol 2004, 338:181-199.

10. Bradford JR, Westhead DR: Improved prediction of protein-protein binding sites using a support vector machines approach. Bioinformatics 2005, 21 (8):1487-1494.

11. Liang S, Zhang C, Liu S, Zhou Y: Protein binding site prediction using an empirical scoring function. Nucl Acids Res 2006, 34(13):3698-3707.

12. Li N, Sun Z, Jiang F: Prediction of protein-protein binding site by using core interface residue and support vector machine. $B M C$ Bioinformatics 2008, 9:1-13.

13. Qin S, Zhou HX: meta-PPISP: a meta web server for protein-protein interaction site prediction. Bioinformatics 2007, 23(24):3386-3387. 
14. Huang B, Schröder M: Using protein binding site prediction to improve protein docking. Gene 2008, 422:14-21.

15. Porollo A, Meller J: Prediction-based fingerprints of protein-protein interactions. Proteins 2007, 66(3):630-645.

16. Chen $R, L i L$, Weng Z: ZDOCK: an initial-stage protein-docking algorithm. Proteins 2003, 52:80-87.

17. Heifetz A, Katchalski-Katzir E, Eisenstein M: Electrostatics in protein-protein docking. Protein Sci 2002, 11(3):571-587.

18. Schneidman-Duhovny D, Inbar Y, Nussinov R, Wolfson HJ: Geometry-based flexible and symmetric protein docking. Proteins 2005, 60(2):224-231.

19. Fernández-Recio J, Totrov M, Skorodumov C, Abagyan R: Optimal docking area: A new method for predicting protein-protein interaction sites. Proteins 2005, 58(1):134-143.

20. Dominguez $C$, Boelens R, J BAMJ: HADDOCK: a protein-protein docking approach based on biochemical or biophysical information. J Am Chem Soc 2003, 125:1731-1737.

21. Alcaro S, Gasparrini F, Incani O, Caglioti L, Pierini M, Villani C: Quasi flexible automatic docking processing for studying stereoselective recognition mechanisms, part 2: Prediction of DeltaDeltaG of complexation and 1H-NMR NOE correlation. J Comput Chem 2007, 28(6):1119-1128.

22. Fernández-Recio J, Totrov M, Abagyan R: Identification of protein-protein interaction sites from docking energy landscapes. J Mol Biol 2004, 335(3):843-865.

23. Zhang C, Vasmatzis G, Cornette JL, DeLisi C: Determination of atomic desolvation energies from the structures of crystallized protein. J Mol Biol 1997, 267(3):707-726.

24. Zhang $C$ : Extracting contact energies from protein structures: a study using a simplified model. Proteins 1998, 31(3):299-308.

25. Li SC, Bu D, Xu J, Li M: Finding largest well-predicted subset of protein structure models. In Combinatorial Pattern Matching, Volume 5029 of Lecture Notes in Computer Science. Edited by Ferragina P, Landau G. Berlin / Heidelberg: Springer; 2008:44-55.

26. Huang B, Schröder M: LIGSITE ${ }^{\text {CSC }}$ : Predicting ligand binding sites using the connolly surface and degree of conservation. BMC Struct Biol 2006, 6:19-29.

27. Henrich S, Salo-Ahen OM, Huang B, Rippmann FF, Cruciani G, Wade RC: Computational approaches to identifying and characterizing protein binding sites for ligand design. $J$ Mol Recognit 2010, 23:209-219.

28. Smith TF, Waterman MS: Identification of common molecular subsequences. J Mol Biol 1981, 147(1):195-197.

29. Liu S, Gao Y, Vakser I: Dockground protein-protein docking decoy set. Bioinformatics 2008, 24:2634-2635.

30. Mintseris J, Wiehe K, Pierce B, Anderson R, Chen R, Janin J, Weng Z: Protien-protein docking benchmark 2.0: an update. Proteins 2005 60:214-216.

31. Janin J, Henrick K, Moult J, Eyck L, Sternberg M, Vajda S, Vakser I, Wodak S: CAPRI: A critical assessment of predicted interactions. Proteins 2003, 52:2-9

32. Hwang H, Pierce B, Mintseris J, Janin J, Weng Z: Protein-protein docking benchmark version 3.0. Proteins 2008, 73:705-709.

33. Hwang $\mathrm{H}$, Vreven $\mathrm{T}$, Janin J, Weng Z: Protein-protein docking benchmark version 4.0. Proteins 2010, 78:3111-3114.

34. Grünberg R, Leckner J, Nilges M: Complementarity of structure ensembles in protein-protein binding. Structure 2004, 12(12):2125-2136

35. Chen $R$, Li L, Weng Z: ZDOCK: An initial-stage protein-docking algorithm. Proteins 2003, 52:80-87.

36. Smith GR, Sternberg MJ: Evaluation of the 3D-Dock protein docking suite in rounds 1 and 2 of the CAPRI blind trial. Proteins 2003, 52:74-79.

37. Carter P, Lesk VI, Islam SA, J SM: Protein-protein docking using 3D-Dock in rounds 3, 4, and 5 of CAPRI. Proteins 2005, 60:281-288.

doi:10.1186/1471-2105-13-158

Cite this article as: Guo et al:: Protein-protein binding site identification by enumerating the configurations. BMC Bioinformatics 2012 13:158.

\section{Submit your next manuscript to BioMed Central} and take full advantage of:

- Convenient online submission

- Thorough peer review

- No space constraints or color figure charges

- Immediate publication on acceptance

- Inclusion in PubMed, CAS, Scopus and Google Scholar

- Research which is freely available for redistribution 